\title{
Waiting for Landauer
}

\author{
John D. Norton ${ }^{1}$ \\ Department of History and Philosophy of Science \\ Center for Philosophy of Science \\ University of Pittsburgh \\ http://www.pitt.edu/ jdnorton
}

\begin{abstract}
Landauer's Principle asserts that there is an unavoidable cost in thermodynamic entropy creation when data is erased. It is usually derived from incorrect assumptions, most notably, that erasure must compress the phase space of a memory device or that thermodynamic entropy arises from the probabilistic uncertainty of random data. Recent work seeks to prove Landauer's Principle without using these assumptions. I show that the processes assumed in the proof, and in the thermodynamics of computation more generally, can be combined to produce devices that both violate the second law and erase data without entropy cost, indicating an inconsistency in the theoretical system. Worse, the standard repertoire of processes selectively neglects thermal fluctuations. Concrete proposals for how we might measure dissipationlessly and expand single molecule gases reversibly are shown to be fatally disrupted by fluctuations. Reversible, isothermal processes on molecular scales are shown to be disrupted by fluctuations that can only be overcome by introducing entropy creating, dissipative processes.
\end{abstract}

${ }^{1}$ I thank Dan Parker for prompting me to look at LPS and for helpful discussion; Owen Maroney for helpful discussion on an earlier draft of this note; and an anonymous referee for detailed and thoughtful responses to earlier versions. 


\section{Introduction}

About fifty years ago, Rolf Landauer (1961) proposed that there is an unavoidable cost in thermodynamic entropy whenever we erase information: the thermodynamic entropy of our surroundings must increase by at least $\mathrm{k} \ln 2$ for each bit of information erased. It was then an interesting speculation supported by a vague plausibility argument. As the decades passed and efforts were put into finding a more precise justification, Landauer's speculation was promoted into "Landauer's Principle." It became a foundational proposition of the new study of the thermodynamics of computation; and it supplanted others to become the favored explanation for the supposedly necessary failure of Maxwell's demon to reverse the second law of thermodynamics.

During the time that its centrality in this new literature was solidified and celebrated, we have continued to wait for a successful justification of Landauer's speculation. Although its absence has been overlooked largely, a small but persistent literature has been drawing attention to the fact that the principle is widely presumed but has no precise grounding. (For this concern and broader concerns over the literature concerning Maxwell's demon, see Earman and Norton (1998, 1999); Shenker (1999), Albert (2000, Ch. 5); Maroney (2005); Norton (2005); ${ }^{2}$ Shenker and Hemmo (2006); and, for a general survey, Maroney (2009).)

There have been attempts to secure more precise demonstrations of Landauer's principle. My attempt (Norton, 2005, Section 2.3) gave a careful demonstration of a version of the principle that depends upon erasure being performed inefficiently. It suggested that the principle depends essentially on a poor choice of a convenient, but dissipative erasure procedure and did not derive from some essential feature of erasure itself. I also noted that arguments for Landauer's Principle repeatedly used the same incorrect assumptions: that erasure must compress phase volume or that additional thermodynamic entropy derives from the probabilities of so-called "random" data. First rank physics journals now offer proofs of Landauer's Principle

\footnotetext{
2 I take this opportunity to correct an error in Norton (2005). The Landauer entropy cost of erasure for $n$ bits was identified as $k \ln n$, whereas it should have been $k \ln N=n k \ln 2$, where $N$ $=2^{\mathrm{n}}$ is the number of states associated with $\mathrm{n}$ bits. The error compromises none of the analysis or results. I am grateful to James Ladyman for pointing out the error.
} 
that employ an impressive repertoire of precise statistical mechanical theory. (See for example, Piechocinska, 2000, and Turgut, 2009.) However, as I shall show in an appendix, these proofs still depend on the same incorrect assumptions as used by earlier, simpler proofs. They are just more deeply hidden.

A notable advance came with the work of Ladyman et al. $(2007,2008)$ (henceforth “LPSG” and "LPS”). LPSG seeks to demonstrate a generalized version of Landauer's Principle without using the incorrect assumptions mentioned above. The sequel, LPS, seeks to demonstrate a corollary with similar rigor: that the information theoretic entropy assigned to a probabilistic mixture of macrostates and commonly associated with "random data" may also have thermodynamic meaning.

The LPSG proof employs a novel strategy. Earlier demonstrations, including my own of 2005, have used what I shall call a "direct" approach. These proceeded by examining directly the physical process of erasure. They automatically illuminate how the supposed entropy cost of erasure arises. LPSG seeks to establish the entropy cost of erasure indirectly. They couple a process that assuredly reduces thermodynamic entropy with an erasure process. They posit independently a statistical form of the second law of thermodynamics. That law then requires that the erasure process must create entropy in order to balance the entropy reduction of the first process.

The attraction of this method is that we can leave the details of the erasure process undefined and seek a result that will apply to all erasure procedures. The weakness is that it automatically precludes illumination of the origin of the entropy cost of erasure; we can only infer that it must be there if the suppositions obtain. And whether they do obtain will prove to be the weakness of the indirect proof. It also means that it cannot explain the supposed failure of a Maxwell demon who seeks to violate the second law of thermodynamics. We consider at the outset only physical systems that, by presumption, will not permit this violation.

In this note I will try to explain why I believe that this new, indirect proof fails. In positing a statistical form of the second law of thermodynamics at the outset, LPSG and LPS make no attempt to ground the law in the underlying physical properties of the systems to be 
investigated. ${ }^{3}$ Rather they presume that that the physical properties of the systems investigated can support a statistical form of the law. Taken in its context, this presumption is mistaken. For the proof also adopts what has tacitly become a standard repertoire of idealized processes from the literature in the thermodynamics of computation to map out the admissible physical systems and processes. The repertoire, which I shall assemble and list explicitly, includes familiar items: single molecules trapped in cylinders fitted with pistons and sliding partitions; and dissipationless processes for locating the position of the molecule. This repertoire enables one to construct processes that assuredly breach even a statistical form of the second law of thermodynamics. Therefore the proof proceeds from inconsistent assumptions. Its methods can be used equally, as I shall show, to demonstrate that erasure can be carried out without entropy cost; or, more precisely, without an assured passing of heat to a surrounding heat bath or some equivalent dissipative process.

These same problems compromise the result of LPS concerning information theoretic entropy, for that analysis uses the same repertoire of processes as well as supposing a statistical form of the second law. In addition, the proof employs a generalized notion of reversibility that is incompatible with thermodynamic entropy being a state property.

It must be stressed that my purpose here is not to assail LPSG and LPS. On the contrary, their efforts are a praiseworthy attempt to bring more secure foundations to the thermodynamics of computation. Rather, the thoroughness of their analyses makes it possible for me to illustrate the sense, already articulated with my colleague John Earman in Earman and Norton (1999), that something has gone seriously awry in this literature.

Sections 2 and 3 below provide an orientation in the thermodynamics of a one-molecule gas and a brief review of the failed efforts to demonstrate Landauer's Principle so far by direct proofs. Section 4 develops the LPSG indirect proof of Landauer's Principle for a special case. Sections 5 and 6 lay out the reasons for the failure of the proof and includes my assembly of a standard inventory of processes from those routinely used in this literature. Section 7 explains how selective neglect of thermal fluctuations compromises the viability of the processes

${ }^{3}$ LPSG, pp. 61-62, write: "We make no assumptions about the relationship between phenomenological thermodynamics and statistical mechanics, but we assume that the second law stated in terms of thermodynamic entropy is valid." 
presumed in this inventory. It demonstrates the fatal disruption by fluctuations of concrete proposals for dissipationless measurement and reversible expansion and compression of a single molecule gas. It also demonstrates a general result that fluctuations preclude reversible, isothermal processes proceeding. Their probability of completion can be raised only by introducing entropy creating disequilibria that are large on molecular scales. Section 8 reviews the LPS result for a special case and the failure of its proof. An Appendix reviews the failure of two recent attempts for direct proofs of Landauer's Principle (Piechocinska, 2000; Turgut, 2009).

\section{Szilard's One-Molecule Gas}

\subsection{Admissible Processes}

The origins of the present literature lie in the recognition over a century ago of the molecular basis of the thermal properties of ordinary matter. That recognition allowed violations of the second law of thermodynamics. A kinetic gas could, with very low probability, spontaneously recompress to a much smaller volume, in contradiction with the second law of thermodynamics. Other thermodynamic fluctuation phenomena, such as Brownian motion, provided less extreme examples of higher probability of the same sort of violation.

In the literature that developed, one particular illustration of these fluctuation effects has come to dominate the theorizing. It is a single molecule of a gas trapped in a cylindrical chamber, whose conception was introduced by Szilard (1929) in his founding paper. It manifests fluctuations in density as the molecule moves about the chamber and fluctuations in pressure through the momentary collisions with the chamber walls. One might imagine that this onemolecule gas is too removed from a many molecule gas to admit ordinary thermodynamic analysis. That suspicion proves wrong. Averaged over time, the successive molecular impacts will smooth out to a pressure on a piston in the chamber that conforms to the ideal gas law. If the piston moves very slowly and the chamber is kept in contact with a heat bath at temperature $\mathrm{T}$, the one-molecule gas may undergo a reversible, isothermal expansion or contraction, governed by the same laws as obtain for ideal gases, as shown in Figure 1. These processes are codified for later reference as: 
Process 1a. Reversible isothermal expansion to double volume. A molecule trapped in one or other half of the chamber by a piston undergoes a reversible, isothermal expansion to full volume. Heat kT $\ln 2$ is drawn from the heat bath and work $\mathrm{kT} \ln 2$ is extracted. Process 1b. Reversible isothermal compression to half volume. A piston reversibly and isothermally compresses the space occupied by the molecule from full to half volume. Heat kT $\ln 2$ is delivered to the heat bath and work kT $\ln 2$ is consumed.
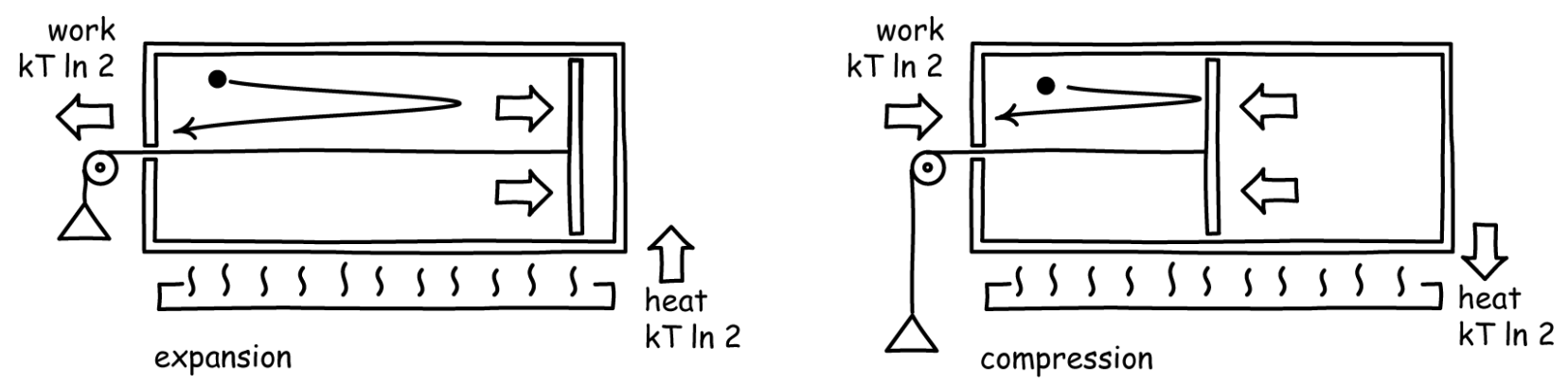

Figure 1. Reversible isothermal expansion and contraction of a one-molecule gas

This expansion and contraction may be achieved more rapidly by two further processes shown in Figure 2:

Process 2a. Removal of the partition. A partition at the midpoint that traps the molecule on one or other side is removed. The removal involves no work or heat Process $2 b$. Insertion of the partition. A partition, inserted at the midpoint of the cylinder, traps the molecule on one or other side with equal probability. The insertion involves no work or heat. 


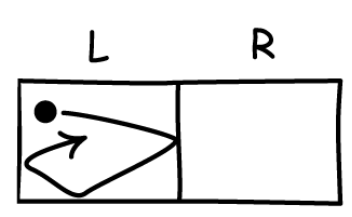

or

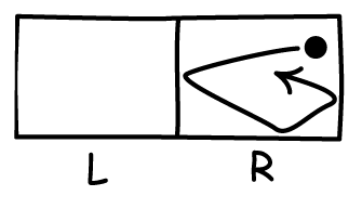

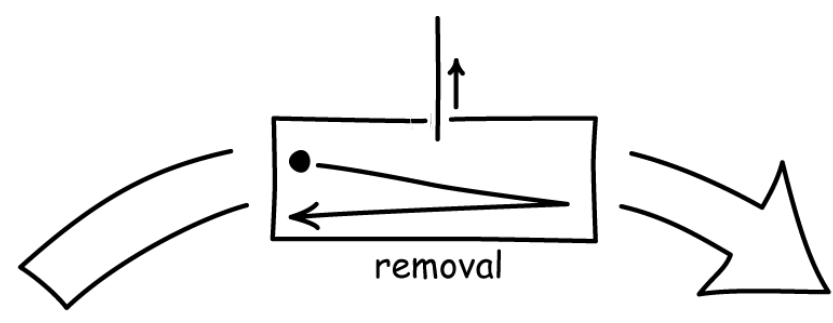

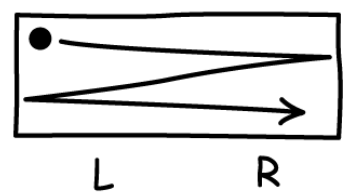

$L$

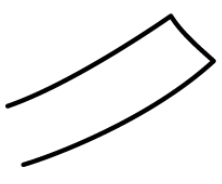

insertion

Figure 2. Removal and Insertion of the Partition

Processes 1a and 2a are analogous in that they both double the volume of the gas; and Process $1 \mathrm{~b}$ and $2 \mathrm{~b}$ both halve the volume of the gas.

\subsection{Thermodynamic Entropy and the Second Law}

We would normally say that the expansion Processes $1 a$ and $2 a$ increase the thermodynamic entropy of the gas by $\mathrm{k} \ln 2$; and that the compression Processes $1 b$ and $2 b$ decrease the thermodynamic entropy by $\mathrm{k} \ln 2$. This follows from an application to Processes $1 a$ and $1 b$ of the Clausius definition of thermodynamic entropy: the difference in thermodynamic entropy $\Delta \mathrm{S}_{\text {therm }}$ for two states of a system is defined by

$$
\Delta \mathrm{S}_{\mathrm{therm}}=\int \mathrm{dq}_{\mathrm{rev}} / \mathrm{T}
$$

where $\mathrm{q}_{\mathrm{rev}}$ is the heat gained by the system during a reversible process connecting the states along which the integration proceeds.

However the circumstances are not normal and we must proceed cautiously. The Clausius formula (1) can only identify thermodynamic entropy $S_{\text {therm }}$ as a property of the initial and final states themselves, if every reversible process that connects the two states gives the same entropy 
difference through formula (1). This sameness can fail if the second law of thermodynamics fails.

The applicable version of the law is the "Thomson" form that prohibits any process whose net effect is just the full conversion of a quantity of heat into work. If we take the special case of two states at the same temperature $\mathrm{T}$ connected by isothermal, reversible processes, one easily sees that this law is necessary if the entropy change of (1) is to depend only on the properties of the initial and final states, so that every isothermal, reversible process connecting them returns the same entropy difference. For, imagine otherwise, that the law fails for a cycle of isothermal, reversible processes on which the initial and final states lie as shown on the left in Figure 3. Then the heats $\mathrm{q}_{1}$ and $\mathrm{q}_{2}$ passed to the system in the two sections of the cycle, process 1 and process 2 , must satisfy $\mathrm{q}_{1}+\mathrm{q}_{2}<0$. Therefore $\mathrm{q}_{1}$ cannot equal $\left(-\mathrm{q}_{2}\right)$. Now consider the initial and final states connected by two processes, process $_{1}$ and the reverse of process $_{2}$, as shown in the figure on the right. The two processes pass unequal heats $q_{1}$ and $\left(-q_{2}\right)$ to the system. Recalling that these are isothermal processes, it follows that the Clausius formula (1) would assign a different entropy difference between the states according to the process that connects them.
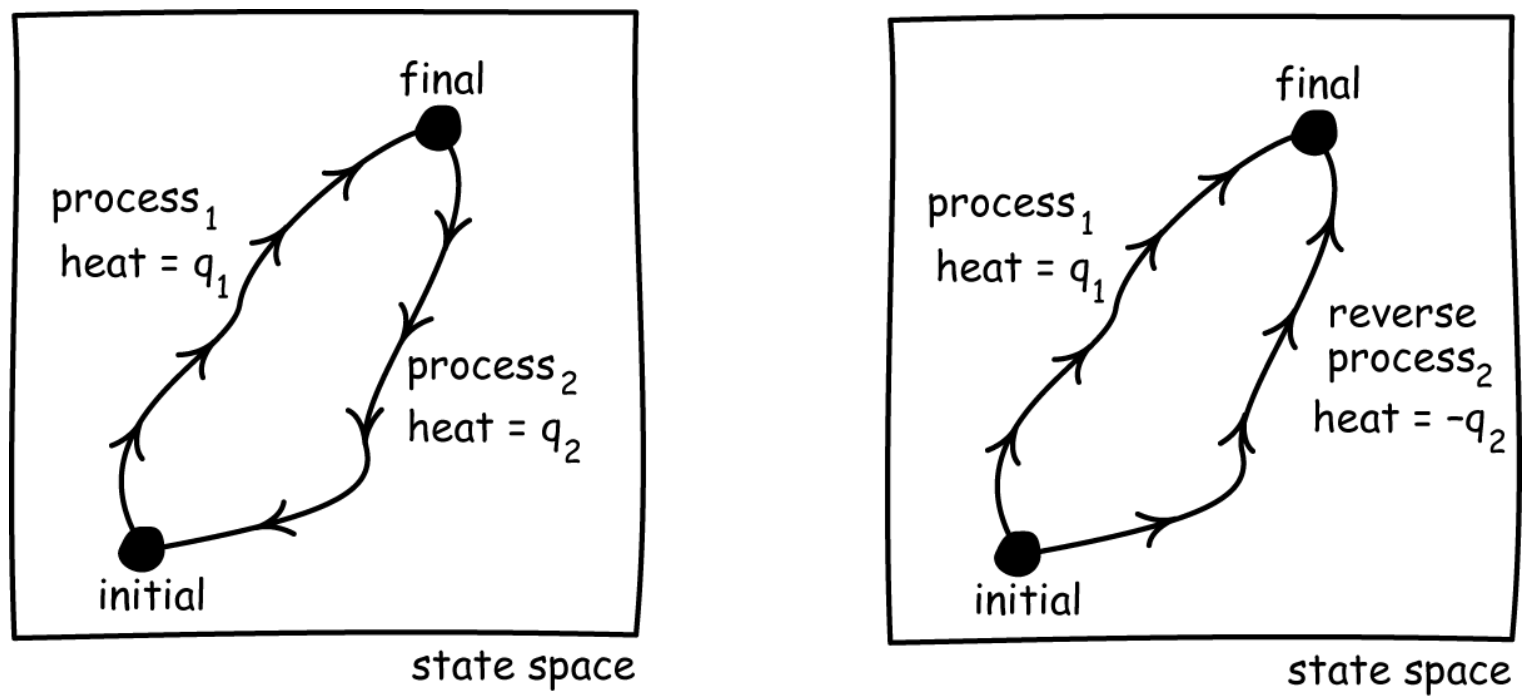

Figure 3. A Cycle that violates the Thomson form of the second law 


\subsection{A Possible Fluctuation-Derived Violation of the Second Law}

Caution is warranted in asserting the second law of thermodynamics, for the random motions of the single molecule, understood as density fluctuations, do constitute continuing violations of the second law. When the molecule moves to one side or other of the chamber, that motion is a spontaneous recompression of the one-molecule gas to one side. All that is needed, it would seem, is the insertion of the partition to make one of the fleeting violations permanent.

More carefully, combining the spontaneous compression of Process $2 b$ with the reversible expansion of Processes la produces a cycle that assuredly violates the second law of thermodynamics. The first step realizes a process forbidden by the second law. The second step converts it into a form that explicitly breaches the Thomson form of the law: kT $\ln 2$ of heat is drawn from the heat bath and converted fully to work, while the gas is returned to its original state. The sole effect of the cycle is to convert heat to work. It is a simple, vivid illustration of the sort of process a Maxwell demon seeks to realize.

One's initial reaction may well be that that we must abandon the second law of thermodynamics if we are to consider fluctuating physical systems such as these; and that may well be the final decision as well. However a literature that is now eighty years old has refused to accept this decision. It seeks to save the second law by finding a hidden locus of entropy creation in the processes of the demon that operates the cycle, now naturalized as a physical device. The earlier tradition, associated most prominently with Brillouin, located the hidden entropy creation in the physical processes used by the demon to identify the position of the molecule. A later tradition deemed this a mistake. Rather, it urged that the demon must retain a memory of which side of the chamber held the molecule. Completing the cycle requires erasure of this memory and, following Landauer, this erasure process creates the entropy needed to preserve the second law.

\section{Direct Proofs of Landauer's Principle}

Applied to the case of one bit of information, Landauer's Principle asserts that its erasure increases the thermodynamic entropy of the surroundings by at least $\mathrm{k} \ln 2$. In Norton (2005), I investigated at some length the approaches used in attempts to prove this principle and explained why they fail. This section will review very briefly the modes of their failure and the reader is 
referred to Norton (2005) for more details. The proofs are direct. That is, they look at the physical process of erasure directly and seek to demonstrate that its implementation must create thermodynamic entropy. The standard model of a memory device is just the one-molecule gas above. A single bit of information is encoded in the trapping of the molecule in the left " $\mathrm{L}$ " or right " $R$ " side of the chamber. Erasure is the process that assuredly moves the molecule to the left chamber L. While this model may seem excessively idealized, it is taken to capture the essential thermodynamic features of a more realistic one-bit memory device in a heat bath. Here are three related attempts to prove Landauer's Principle, along with the reasons they fail.

\subsection{Erasure by Thermalization.}

The molecule is trapped on one side of the partition. In the erasure procedure, the partition is removed by Process $2 a$, thermalizing the molecule. The resulting chamber-filling one-molecule gas is isothermally and reversibly compressed by Process $1 b$ to the reset L state. This step passes heat kT $\ln 2$ to the heat bath at temperature T, increasing its entropy by $\mathrm{k} \ln 2$.

All this proof shows is that a particular, inefficient erasure procedure creates thermodynamic entropy $\mathrm{k} \ln 2$, whose origin lies in the ill-advised initial step of removing the partition. It does not show that all possible erasure processes must create thermodynamic entropy.

It is sometimes suggested that the erasure must employ the thermodynamic entropy creating step of removal of the partition, or something like it, for the process cannot "know" which side holds the molecule on pain of requiring further erasure of that knowledge. Yet the erasure must succeed whichever side holds the molecule. ${ }^{4}$ However what this consideration does not show is that thermalization is the only way of satisfying this robustness condition. Indeed, in light of the analysis below, it cannot be shown in the standard framework, since its repertoire of processes admits other robust erasure procedures that do not employ thermalization.

\subsection{Compression of Phase Volume.}

Boltzmannian statistical physics relates the thermodynamic entropy $\mathrm{S}$ of a system to the accessible volume of the phase space it occupies according to

${ }^{4}$ For an example of this approach, see Leff and Rex (2003, p.21.) 


$$
\mathrm{S}=\mathrm{k} \ln \text { (accessible phase volume) }
$$

Prior to the erasure, the memory device may contain either L or R data. Therefore, this approach asserts, the molecule is associated with a phase volume that spans both chambers. After erasure, the molecule is assuredly in the L half of the chamber, so that the phase volume has been halved. The resulting entropy reduction is $\mathrm{k} \ln 2$. That reduction must be compensated by at least a doubling of the phase volume of the surroundings and, correspondingly, an increase in its entropy of at least $\mathrm{k} \ln 2.5$

The error of the proof is that the molecule, prior to the erasure, is not associated with an accessible phase volume that spans the entire chamber. It will assuredly be in one half only. Which that half is will vary from occasion to occasion, but it will always be one half. As a result, the erasure operation does not need to reduce accessible phase volume at all; it merely needs to relocate the part of phase space accessible to the molecule.

\subsection{Illicit Ensembles and Information Theoretic Entropy.}

For so-called "random data," the molecule is equally likely to be in the L or R chamber. That is, we have probabilities $\mathrm{P}(\mathrm{L})=\mathrm{P}(\mathrm{R})=1 / 2$. After erasure, the molecule is assuredly in the $\mathrm{L}$ chamber. That is, $\mathrm{P}(\mathrm{L})=1 ; \mathrm{P}(\mathrm{R})=0$. In this approach, the "random data" state is treated as if it was the same as a thermalized data state, whose erasure does create $\mathrm{k} \ln 2$ in thermodynamic entropy. We arrive at the thermalized data state from the random data state by Process $2 a$, removal of the partition, so that the molecule can move freely between the two chambers. It is supposed that both states are thermodynamically equivalent, since, in both states the molecule is equally probably in either chamber. The thermodynamic equation of the two states is sometimes justified by considering a large set of memory devices containing random data. This set is

\footnotetext{
5 Writing some three decades after his initial proposal, concerning a figure that shows information degrees of freedom vertically and environmental degrees of freedom horizontally, Landauer (1993, p.2) glosses the result in this way: "The erasure process we are considering must map the 1 space down into the 0 space. Now, in a closed conservative system phase space cannot be compressed, hence the reduction in the vertical spread [of phase volume] must be compensated by a lateral phase space expansion, i.e. a heating of the horizontal irrelevant degrees of freedom, typically thermal lattice vibrations."
} 
presented as a canonical ensemble, supposedly thermodynamically equivalent to a canonical ensemble of thermalized memory devices.

The approach fails because the random data state and the thermalized state are not thermodynamically equivalent. In the random state, only half the phase space is accessible. In the thermalized state, the full phase space of the chamber is accessible to molecule. This difference of accessibility gives the two states different thermodynamic properties. They differ by $\mathrm{k} \ln 2$ in thermodynamic entropy. That we do no know which half of the phase space is accessible in the case of random data is irrelevant to the device's thermodynamic properties. (For further discussion, see Norton, 2005, §3.)

A development of this approach calls on information theory. There one assigns an entropy $S_{\text {info }}$ to a probability distribution that assigns probabilities $P_{i}$ to outcomes $i=1, \ldots n$ according to

$$
\mathrm{S}_{\text {info }}=-\mathrm{k} \sum_{\mathrm{i}} \mathrm{P}_{\mathrm{i}} \ln \mathrm{P}_{\mathrm{i}}
$$

where units of $\mathrm{k}$ are chosen arbitrarily. Applying this formula 6 to the probability distributions before and after erasure, we recover a change in entropy of $k \ln 2$.

Where this argument fails is that it only establishes a change in information theoretic entropy $\mathrm{S}_{\text {info }}$. Without further assumption, it does not establish a change in thermodynamic entropy $S_{\text {therm }}$ as defined by the Clausius formula (1). Thermodynamic and information theoretic entropies have been shown to coincide in the special case in which the probability distribution is a canonical distribution over a volume of phase space that is everywhere accessible to the system point. (The demonstration is given in Norton, 2005, Section 2.2.) This condition of accessibility fails for the probability distribution of random data.

There is a second difficulty with this approach that has not, to my knowledge, been taken up in the literature. The input to an erasure procedure will be a memory device containing an indeterminate configuration of data. In the one bit case, the data to be erased may be $\mathrm{L}$ or R. This

\footnotetext{
6 Invoking this information theoretic entropy, Leff and Rex (2003, p. 21) write of a memory device containing random $\mathrm{L}$ and $\mathrm{R}$ data: “...the initial ensemble entropy per memory associated with the equally likely left and right states is $\mathrm{S}_{\mathrm{LR}}($ initial $)=\mathrm{k} \ln 2 . "$
} 
is routinely modeled by describing the data as "random," thereby adding a probability distribution to the possible data. This introduction of a probability distribution is essential if formula (3) is to be used, for the quantity it computes is a function of that distribution.

The trouble is that the introduction of a probability distribution is not justified by the logical specification of the erasure function; the specification makes no mention of probabilities. It just indicates a function on the set $\{\mathrm{L}, \mathrm{R}\}$ that cannot be inverted. This introduction of probability is routinely assumed benign in physical analysis when some variable has an indeterminate value. It is not benign since it adds non-trivial structure to the indeterminateness of a variable and can induce egregious inductive fallacies, as shown in Norton (2010). The real seat of the entropy of formula (3) is this probability distribution and, absent cogent justification of the introduction of the probability distribution, ${ }^{7}$ the entropy change associated with erasure by formula (3) is merely an artifact of a misdescription of the indeterminateness of data.

Attempts at direct proofs of Landauer's Principle in the literature employ one or more of these three approaches and, as a result, fail. Two such failed attempts from more recent literature are reviewed in the Appendix.

\section{An Indirect Proof of Landauer's Principle}

The erasure process considered above takes a memory device that may hold either L or R as data and resets it to L. This is a physical implementation of a logical transformation that maps either of the symbols L or R always to L. It is logically irreversible in the sense that the function is not invertible. Informally, knowing the result is $\mathrm{L}$ does not tell us whether an $\mathrm{L}$ or an $\mathrm{R}$ was reset. Landauer's Principle asserts that the associated physical erasure process must create k $\ln 2$ of thermodynamic entropy. As a result, LPSG call the process "thermodynamically irreversible." LPSG seek to establish a generalized form of Landauer's Principle according to which all physical implementations of logically irreversible processes are thermodynamically irreversible.

For present purposes, there is no need to recount LPSG's result and proof in all generality. It will be sufficient to rehearse it for the simple case of one bit erasure seen so far. The proof employs two systems: a memory device $\mathrm{M}$ and a one-molecule gas $\mathrm{G}$, used as a randomizer. We will use a second one-molecule gas as the memory device M. Its initial state will

\footnotetext{
7 Those tempted to call upon a "principle of indifference" should see Norton $(2008,2010)$.
} 
be the molecule in the left side of the chamber, state " $\mathrm{M}_{\mathrm{L}}$," and it will record one bit of information according to the final position of the molecule, $\mathrm{M}_{\mathrm{L}}$ or $\mathrm{M}_{\mathrm{R}}$, with the latter state defined analogously. The process of interest is the erasure process that returns the memory device to its initial state $\mathrm{M}_{\mathrm{L}}$. The proof proceeds by embedding that erasure process in the following thermodynamic cycle, where the step numbering coincides with the step numbering of LPSG's more general proof:

Step 1. The one-molecule gas of G occupies the full chamber. A partition is inserted at the midpoint so the molecule is trapped on one or other side of the chamber with equal probability.

Step 2. The location of the molecule is measured and the memory device $\mathrm{M}$ is set to $\mathrm{L}$ or $\mathrm{R}$ according to whether the molecule of $\mathrm{G}$ is found in the $\mathrm{L}$ or $\mathrm{R}$ side of its chamber. (Since the initial state is $\mathrm{M}_{\mathrm{L}}$, action is only triggered if the molecule is found to be on the right, in which case it will be shifted to the left.) The shift is performed by a reversible thermodynamic process. Since the thermodynamic entropies of $\mathrm{M}_{\mathrm{L}}$ and $\mathrm{M}_{\mathrm{R}}$ are the same, no heat passes to or from the surroundings.

Step 3. According to whether the memory device is in state $\mathrm{M}_{\mathrm{L}}$ or $\mathrm{M}_{\mathrm{R}}$, a piston is introduced into the chamber of $\mathrm{G}$ and a reversible isothermal expansion in direction $\mathrm{R}$ or $\mathrm{L}$ performed, returning the system $\mathrm{G}$ to its initial state. Heat $\mathrm{kT} \ln 2$ is drawn from the heat bath and work kT $\ln 2$ is recovered.

Step 4. The erasure process is performed. It transforms the memory device from the probabilistically mixed state of $\mathrm{M}_{\mathrm{L}}$ or $\mathrm{M}_{\mathrm{R}}$ with equal probability, to the initial state of $\mathrm{M}_{\mathrm{L}}$.

Completion of the analysis requires that we postulate a statistical version of the second law of thermodynamics. It is given by LPSG (p. 65, emphasis in LPSG) as: 
It is impossible to perform a cyclic process with no other result than that on average heat is absorbed from a reservoir, and work is performed.

Steps 1-4 have returned both M and G to their initial states. Step 2 has drawn kT $\ln 2$ heat from the heat bath and converted it into work. Steps 1 and 3 perform no heat/work conversions. Therefore it follows from the statistical version of the second law that, on average, the erasure process of Step 4 must degrade kT $\ln 2$ of work into heat. This is the main result of LPSG in this case: that the erasure of Step 4 is thermodynamically dissipative.

LSPG are quite explicit in asserting that the proof depends on the postulation of the statistical form of the second law (p. 59):

...we do not regard [Landauer's Principle] as more fundamental than the second law of thermodynamics, and so we do not follow those authors who try to show that Landauer's Principle implies the impossibility of a Maxwell demon. Rather, we assume that second law and show that Landauer's Principle follows. Hence we follow the 'sound' rather than the 'profound' horn of the dilemma that Earman and Norton $(1998,1999)$ identified.

\section{Failure of the Indirect Proof}

This proof fails because of this dependence on the postulation of the second law. The repertoire of admissible processes is sufficiently broad to allow composite processes that violate the second law of thermodynamics, even in its statistical form. So one cannot conjoin the law to a system that includes this repertoire without inducing inconsistency.

A difficulty with this proof is that the repertoire of admissible processes is not listed explicitly. Rather LSPG follow the practice of the literature in assuming tacitly that certain primitive processes possible and assembling composite processes from them. We can list a subset of these admissible processes by collecting those used in LSPG's proof. That list was begun in Section 2 and is continued below to form what I shall call the "standard inventory." This inventory seems to be widely, if tacitly, used. ${ }^{8}$

\footnotetext{
8 Turgut (2009) is distinctive for its rich use of the statistical physics of Hamiltonian systems in its proof of its Theorem 1 . The proof of its theorem 2, however, proceeds entirely with processes in the inventory introduced as primitive. A "Szilard's one-molecule gas" in a chamber is
} 


\subsection{Inventory of Admissible Processes}

Step 2 of LPSG's proof allows:

Process $3 a$. Detection. Whether the molecule is trapped by the partition in the left or right half of the chamber can be ascertained by a measurement process that passes no heat to the heat bath.

That this detection proceeds dissipationlessly is important to Process 1a. Reversible isothermal expansion to double volume. For it is routinely assumed in this literature that one cannot undertake Process $1 a$ unless the operator already knows which half of the chamber holds the molecule. ${ }^{9}$ Hence any composite process that seeks to effect this doubling in volume can employ Process $1 a$ without further dissipation, for, if the position of the molecule is not known, one simply inserts Process 3 a. Detection as the immediately prior step.

From Step 2 and Step 3, we see that detection can also be used to initiate other processes conditionally

Process 3b. Detect and Trigger. According the whether the outcome of a detection is L or $\mathrm{R}$, $\operatorname{process}_{\mathrm{L}}$ or $\operatorname{process}_{\mathrm{R}}$ respectively may be initiated, without the initiation passing heat to the heat bath, where these are any two admissible processes.

Finally, from the setting of the memory device M in Step 2 we have:10

manipulated by the insertion and removal of walls and by the thermodynamically reversible expansion and compression of its compartments.

${ }^{9}$ I presume that, for this reason, Step 3 requires the antecedent measuring of the state of the memory device $\mathrm{M}$ before the gas expansion proceeds, since the memory device records the location of the gas molecule of $\mathrm{G}$.

10 This step is a special case of a step from the full proof of LPSG (p. 66). In their step 2(a), a memory device $\mathrm{D}$ a memory device of otherwise unspecified constitution is allowed to have states $\mathrm{D}_{\text {out }}(\mathrm{y})$ and $\mathrm{D}_{\text {in }}\left(\mathrm{x}_{1}\right)$ with corresponding entropies $\mathrm{S}_{\text {out }}(\mathrm{y})$ and $\mathrm{S}_{\mathrm{in}}\left(\mathrm{x}_{1}\right)$ and transforming from the first to the second of these states is associated with a passing of heat to the surroundings of $\mathrm{T}\left(\mathrm{S}_{\text {out }}(\mathrm{y})-\mathrm{S}_{\mathrm{in}}\left(\mathrm{x}_{1}\right)\right)$. 
Process 4 . Shift. If a system has states $\mathrm{M}_{1}$ or $\mathrm{M}_{2}$ of equal thermodynamic entropy, then a shift process moves the system from one state to the other without passing heat to the heat bath.

The full repertoire is the set of Processes $1 a, 1 b, 2 a, 2 b, 3 a, 3 b$ and 4 .

\subsection{A Process that Violates the Second Law of Thermodynamics}

It is straightforward to check that the Steps 1-4 of the proof of Section 4 employ processes from the inventory of Section 5.1. It is equally straightforward to check that we can devise an alternative to Step $4^{*}$ that also only employs admissible processes: ${ }^{11}$

Step $4^{*}$. Dissipationless erasure. If the memory device is measured to be in state $\mathrm{M}_{\mathrm{R}}$, a shift process is initiated that moves the molecule from state $M_{R}$ to state $M_{L}$ with equal thermodynamic entropy by a process that passes no heat to the heat bath.

This alternative step employs Process 3b. Detect and Trigger. and Process 4. Shift. The shifting of state $\mathrm{M}_{\mathrm{R}}$ to state $\mathrm{M}_{\mathrm{L}}$ is the same shift as described in Step 2. One can readily conceive processes akin to those already employed that would achieve it. For example, the molecule might be enclosed in a box that is slowly moved from its position in the right of the chamber to the left, as shown in Figure 4.

11 The inclusion of a new detection process in Step $4^{*}$ is not the most efficient implementation, but is convenient for exposition. One could merge Step 3 and Step $4 *$ so that the detection process of Step 3 is all that is needed. If Step 3 detects $M_{R}$, for example, it initiates a single process that both expands the gas of $G$ and then resets state $M_{R}$ to state $M_{L}$. A further economization would employ only the detection operation of Step 2. 

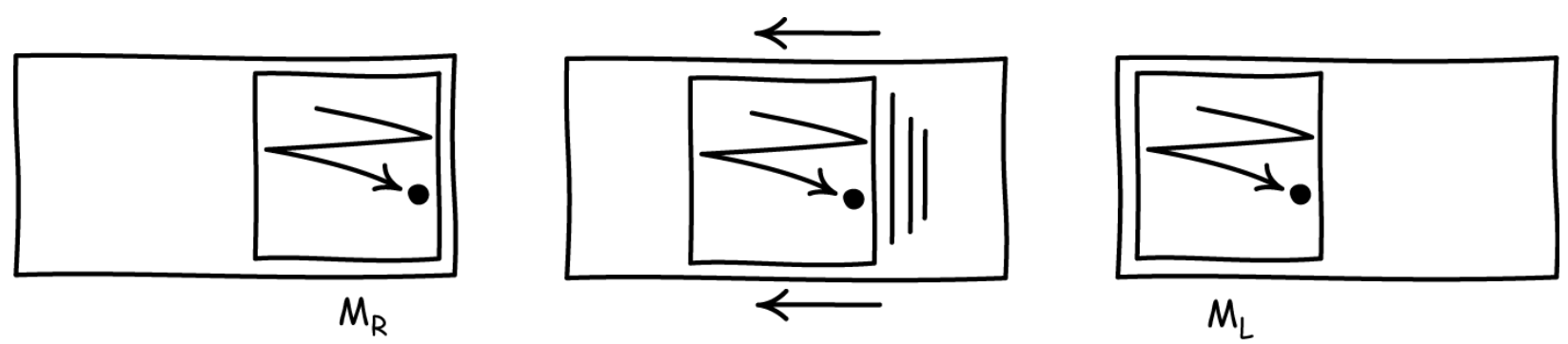

Figure. Shifting a Memory Device from State $M_{R}$ to State $M_{L}$

The net effect of a process with Steps $1,2,3,4^{*}$ is the return of the one-molecule gas $\mathrm{G}$ to its initial, fully expanded state, the return of the memory device $M$ to its initial state $M_{L}$ and the full conversion of heat kT $\ln 2$ to work, in direct contradiction with the Thomson form of the second law of thermodynamics. Since the cycle can be repeated indefinitely, it also contradicts the statistical form of the law.

As the labeling of Step $4^{*}$ indicates, the step by itself embodies a dissipationless erasure process, in contradiction with the assertion of Landauer's principle. While the repertoire is limited, it is not the only dissipationless erasure process admitted by this repertoire of processes. Here is another. The goal is to reset the memory device, provided in state $M_{R}$ or state $M_{L}$, back to its neutral state $\mathrm{M}_{\mathrm{L}}$. The process detects whether the molecule is trapped in the right side of the chamber. If it is, the partition is removed and replaced. This detect-remove-replace process is repeated until the molecule is no longer found in the right side. The probability rapidly approaches one that the erasure is complete upon repetition of the detect-remove-replace process. (There is a probability greater than 0.999 of shifting of the molecule from R to L after 10 repetitions.)

\section{Dissipationless Erasure and the No-erasure Demon}

The second law violating process comprised of Steps $1,2,3,4^{*}$ is not new. It just a version of the "no-erasure" demon described in Earman and Norton (1999, pp. 16-17) and Norton (2005, pp.404-405). (The label "no erasure" was used to indicate only that the process does not perform a dissipative erasure of the type traditionally described.) Proponents of Landauer's Principle have challenged this demon. However, I believe that the challenges have failed. 
The dissipationless erasure processes described here and in the no-erasure demon are not intended as positive proposals. Rather they are introduced as the final stages of a reductio argument. That argument seeks to establish the pointlessness of trying to establish Landauer's Principle or the necessary failure of Maxwell demon in a system that employs processes listed here. For those processes admit construction of both dissipationless erasure routines and a Maxwell demon.

\subsection{Challenge from an Augmented Landauer Principle}

Bennett (2003) suggested that the no-erasure demon is subject to an extended form of Landauer's Principle. The no-erasure demon must merge computational paths in order to restore the gas and memory device to their original states. This merging, the augmented principle states, is accompanied by a compensating entropy increase in the surroundings. In Norton (2005, Section 5), I have explained in more detail why this augmentation of Landauer's Principle fails to compromise the no-erasure demon. Briefly, the notion of computational path and the computational space it suggests is vague, making any precise determination of the grounding of the claim unclear. The intention seems to be that this merging must create entropy in the same way as the failed "reduction of phase volume" argument of Section 3.2 for the unaugmented Landauer's Principle. There is no reason to expect this vaguer rendering of a failed argument to fare any better.

Finally, the no-erasure demon is constructed from the inventory of admissible processes routinely presumed in the thermodynamics of computation. Bennett's response does not dispute that. Hence his challenge merely worsens the mismatch of Landauer's Principle with the standard inventory by expanding the range of processes admitted by the inventory but prohibited by the now extended Landauer's Principle.

\subsection{Challenge from the Notion of A Control Bit}

LPSG (p. 72, n.8) have suggested the no-erasure demon fails for different reasons. They write

[the no-erasure demon] does not really achieve the implementation of a logically irreversible process because, since the same bit cannot be both the control and the target of a controlled operation, the system must have a 'memory' (our M) of 
which program it has run, which amounts to the system keeping a copy of the original bit; hence, in our terminology it implements [a logically reversible transformation] $\mathrm{L}_{2}$.

The claim, apparently, is that a detection process conducted on a memory device cannot be used to trigger a process that alters that same memory device, unless some record is left elsewhere of the content of the memory device. The claim is unsustainable. The triggered process can proceed without the continued existence the triggering data; all that it needs is for the data to exist at the time of the triggering and the presumption that the processes can proceed independently once triggered. If it helps, imagine that the process triggered is carried out by a physically distinct robotic machine. The device's sole function is to perform this one process without needing any further data input; it operates autonomously once triggered; and it is programmed to return itself to its unique ready state as its last step.

\section{Arbitrariness of the Standard Inventory}

In Section 5, I assembled what I called the standard inventory of process employed in this literature. I argued that this inventory admits composite processes that violate the second law of thermodynamics and also effect dissipationless erasure. My point is not to urge that such processes are possible. Rather my point is one that has been central to my earlier papers on the subject: that the literature in the thermodynamics of computation is incoherent. Its basic principles--versions of the second law of thermodynamics and Landauer's Principle--contradict the processes it admits.

I have no interest in defending the standard inventory. My view is that it has been assembled myopically in order to enable a few favored composite processes to be constructed, but without proper attention to the fuller ramifications of the selection. It can be challenged and should be.

\subsection{Neglect of Fluctuations}

The principal difficulty for this inventory comes from its selective neglect of fluctuation phenomena. This neglect reflects the discarding of an earlier tradition in which fluctuations played a central role. When the molecular basis of thermodynamics was accepted over a century ago, fluctuation phenomena were recognized to be small violations of the second law of 
thermodynamics. The open question was whether these small violations could be accumulated into larger ones. The early consensus was that they could not. The operation of devices that sought to accumulate them, it was decided, would be disrupted fatally by fluctuations within their components.

This view was elaborated in the 1910s by Marian Smoluchowski through many examples. One is well known. One might try to realize something close to the demon system Maxwell originally imagined by placing a spring-loaded trapdoor over a hole in a wall separating two gases, each initially at the same temperature and pressure. Molecular collisions can open the trapdoor in one direction only, as shown in Figure 4, to allow molecules to pass from left to right only, creating a disequilibrium in pressure that violates the second law.

The proposal fails because the trapdoor will itself carry thermal energy, fluctuating around the equipartition mean of kT/2 per degree of freedom. Since it must be light-weight enough for a collision with a single molecule to open it, its thermal energy will lead the trapdoor to flap about wildly and allow molecules to pass in both directions. (For a short survey, see Earman and Norton, 1998, pp. 442-48.)

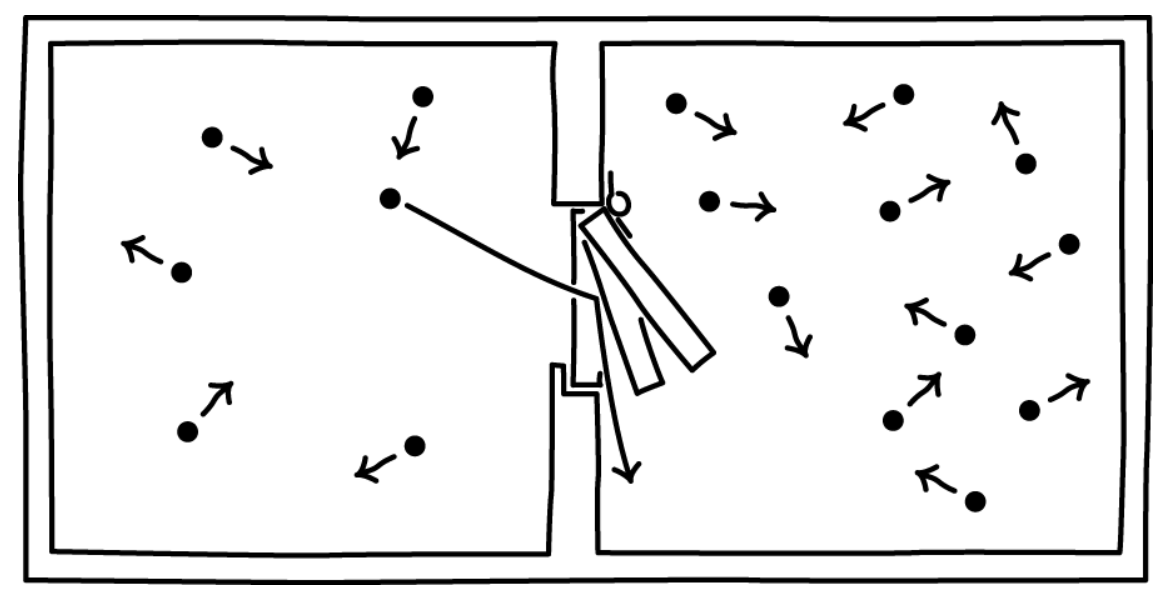

Figure 4. Smoluchowski Trapdoor

\subsection{Reversible, Isothermal Expansion and Contractions}

Fluctuations will disrupt processes in the inventory of Section 5.1. Consider the pair, Process 1a. Reversible isothermal expansion to double volume. and Process $1 \mathrm{~b}$. Reversible isothermal compression to half volume. They are carried out by a piston connected by linkages to 
a weight. Work is extracted from or converted to the thermal energy of the molecule when its collisions with the piston lead it to raise or lower the weight. If the process is to approximate a reversible process, the mean force exerted by the molecular collisions must almost exactly be matched by the mean force of the piston and weight, so the system is as close as possible to a delicate equilibrium of forces. Now each component of the system will have its own mean thermal energy of kT/2 per degree of freedom, comparable in magnitude to the energy of the molecule. That includes the piston, the weight and each component of the linkages that connects them. As a result the entire system of molecule, piston and weight will be bouncing wildly to and fro. If the very slight disequilibrium of forces favors expansion and the raising of the weight, that expansion will not be realized stably. For fluctuation motions will be superimposed upon it, so that the statistical equilibrium state will consist of random motions through some portion of the cylinder. The same holds for compression.

A simple but revealing implementation of the process arises when the cylinder, carrying the single molecule, is oriented vertically with the piston resting on the gas pressure, as shown in Figure 5. The work of expansion and compression arises in the raising and lowering of the weight of the piston by the gas pressure. If the process is to be reversible, the piston must have just enough mass so that, absent fluctuations, the expansion or compression sought is only just favored by the balance of forces. ${ }^{12}$ That means that we will have a light molecule repeatedly colliding with a very light piston and that very light piston will have thermal motions comparable to those of the molecule. As a result, the equilibrium state for the piston will consist of random motions spread over the cylinder.

12 This condition will require adjustments to the mass of the piston as the one-molecule gas expands and contracts and the gas pressure changes. While machinery that adjusts the mass is possible, it will greatly complicate analysis. A better approach is to replace the gravitational field by another force field that pulls the piston down with a force $-2 \mathrm{kT} / \mathrm{h}$, as developed in Section 7.5 below. We also presume that this force does not act upon the molecule, thereby avoiding the complication of a gravitationally induced pressure gradient. 


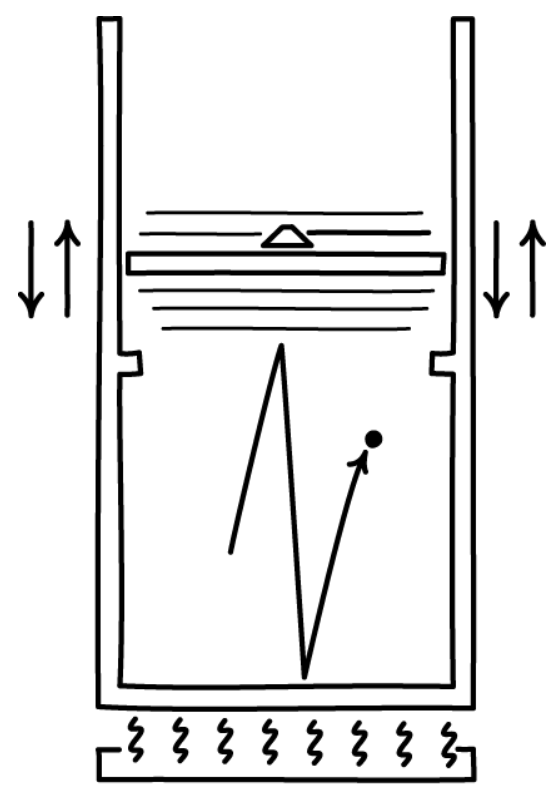

Figure 5. A Piston Reversibly Compressing a One-Molecule Gas

That thermal fluctuations in the piston's position will be of the size of the cylinder as a whole follows from a rough estimate of the size of the thermal fluctuations in the piston's position. Consider the piston as a thermal system at temperature $\mathrm{T}$, independently of the single molecule of the gas. Its energy will be canonically distributed in conformity with the Boltzmann distribution. The distribution of its energy of height $\mathrm{E}=\mathrm{Mgh}$, for height $\mathrm{h}$ above the cylinder floor and acceleration due to gravity g, will scatter the piston thermally over different heights according to the probability density

$$
\mathrm{p}(\mathrm{h})=(\mathrm{Mg} / \mathrm{kT}) \exp (-\mathrm{Mgh} / \mathrm{kT})
$$

for $\mathrm{h} \geq 0$. The mean of this distribution is $\mathrm{kT} / \mathrm{Mg}$ and its standard deviation is also $\mathrm{kT} / \mathrm{Mg}$. This standard deviation $\mathrm{kT} / \mathrm{Mg}$ measures the scale for the size of thermal fluctuations in height of the piston. The mass $\mathrm{M}$ is chosen so that the weight of the piston, $\mathrm{Mg}$, exactly balances the force from the pressure $\mathrm{P}=\mathrm{kT} / \mathrm{V}$ exerted by the one-molecule gas, when it occupies volume V.13 The force exerted on the piston by the gas pressure is just PA, where A is the area of the piston, so that $\mathrm{V}=\mathrm{hA}$. The equilibrium height $\mathrm{h}_{\mathrm{eq}}$ of the piston is determined by setting these two forces equal: $\mathrm{Mg}=\mathrm{PA}=\left(\mathrm{kT} / \mathrm{h}_{\mathrm{eq}} \mathrm{A}\right)$. A. Solving

13 The fuller analysis of Section 7.5 will show that this is an inexact condition for equilibrium. It neglects the effects of the piston's thermal motion on the mean force it exerts on the gas. 


$$
\mathrm{h}_{\mathrm{eq}}=\mathrm{kT} / \mathrm{Mg}
$$

Hence the scale of thermal fluctuations in the position of the piston will be of the size of the displacement of the piston sought in doubling or halving of the cylinder volume.

One might try to arrest fluctuating motions of the compression process at the midpoint or the expansion process at full volume by including a stop that obstructs further motion of the piston. However in both cases the piston will simply bounce off the stop and continue its random motion. One might try to arrest the motion in a more sophisticated way. Perhaps one locates a spring-loaded pin in the wall of the chamber that will release and lock the piston as it passes. We assume our components are governed by a non-dissipative Hamiltonian mechanics that has no friction term. Therefore the pin and spring system will have its own thermal energy and the pin will bounce in and out in a way that defeats its purpose of arresting the piston. We are now replicating Feynman's et al.’s $(1963, \S 46)$ famous analysis of the ratchet and pawl that itself lies within the Smoluchowski tradition.

Finally, even if we could arrest the motion, it is only by chance that the arrested motion would have realized the reversible process sought. The piston may arrive at the end of the cylinder as a result of being raised by work done by the gas pressure. But it may also arrive there through a random fluctuation.

\subsection{Dissipationless Detection}

A second casualty of the neglect of fluctuations is Process $3 a$. Detection. It is presumed possible without dissipation, thereby enabling the localization of the entropy costs of computation into the erasure process. This presumption contradicts an earlier tradition that asserted a necessary entropy cost of $\mathrm{k} \ln 2$ for any measurement that ascertained one bit of information, such as the position of a molecule distributed over two chambers. The history of this tradition was recounted in Earman and Norton (1999, especially Section 2.1), where the assertion of this entropy cost was called "Szilard's Principle." The newer tradition asserted that the earlier tradition was mistaken. However the proposals for procedures that could measure without entropy cost were defective. Their operation would be disrupted fatally by fluctuation processes. (See Earman and Norton, 1999, pp. 13-14; p. 16.)

One celebrated example was offered by Bennett (1987, p.14). To determine which side of a divided chamber holds a molecule, a keel shaped device is slowly lowered onto the chamber. It 
is connected by two pistons to the two sides of the chamber, so that, as it is lowered, one piston's motion is resisted by the single molecule's gas pressure. This tips the keel and reveals the molecule's position. The difficulty is that the keel is a thermal system with mean thermal energy $\mathrm{kT} / 2$ per degree of freedom. It must be very light if the pressure of a single molecule can tip it. The process of lowering the keel onto the chamber is quite similar to the compression of a onemolecule gas by a weight described in the last section. Hence, its thermal energy will cause the keel to rock wildly, just as comparable thermal energies are able to scatter the molecule through the chamber. The resulting rocking motion will preclude the keel from settling into a configuration that reveals the molecule's position.

In another example, Bennett (1982, p. 307-309) describes a dissipationless measurement performed by a ferromagnet. It is initially in a "soft mode" so that it can transform freely between the "up" and "down" states. It couples with the system to be measured and adopts that state's up or down mode. On manipulating the external magnetic field, the ferromagnet reverts to a bistable state in which it can no longer transform freely between the up and down states, completing the measurement process. Once again fluctuations will disrupt the measurement. Each of its steps must be performed reversibly, so they are, at every stage, in a delicate equilibrium upon which fluctuations are superimposed. The part of the detection process that couples the detector ferromagnet to the target data ferromagnet is familiar. It is a two-to-one compression of the magnetic degrees of freedom of the detector space. Hence it is thermodynamically similar to the compression of a one-molecule gas by a piston. This suggests that this coupling process will be disrupted in an analogous way. More generally, since all the processes of the detection are isothermal, reversible processes, we can know that fluctuations will disrupt them, for the general result of the next section applies to them.

\subsection{A General Result: Fluctuation Disruption of All Reversible, Isothermal Processes at Molecular Scales}

The processes of the last two sections admit a simple, general characterization. Each is an isothermal, reversible process in a system at thermal equilibrium with its surroundings. During this process, the system may exchange heat with its surroundings, but it exchanges no work with the surroundings. In the case of the reversible expansions and contractions of Section 7.2, the system consists of the totality of the gas and the machinery that gains or supplies work, such as 
the raised or lowered, weighted piston. In the case of dissipationless detection of Section 7.3, the system consists of a target whose state is measured, a detector and a driver, which couples and decouples the detector from the target.

No process with this general characterization can proceed reversibly on molecular scales; its operation is fatally disrupted by fluctuations. Here is the demonstration. Throughout, the system is in thermal equilibrium at temperature T. Hence, it is canonically distributed over a phase space admitting all its possible configurations. Its probability density is

$$
\mathrm{p}(\mathbf{x}, \boldsymbol{\pi})=\exp (-\mathrm{E}(\mathbf{x}, \boldsymbol{\pi}) / \mathrm{kT}) / \mathrm{Z}
$$

$\mathrm{Z}$ is the normalizing partition function. The multi-component $\mathbf{x}$ and $\pi$ are generalized configuration and momentum coordinates. They may be familiar positions and momenta. But they may equally be canonical quantities associated with the magnetization of the ferromagnet detector and target of the last section.

We will take $\lambda$ to parameterize the stage of the process, which starts at $\lambda=\lambda_{1}$ and ends at $\lambda=\lambda_{2}$. The different stages $\lambda$ of the process correspond to different subvolumes of this phase space. For example, an initially compressed state of a one-molecule gas will correspond to a subvolume of the phase space in which the molecule accesses a portion of its configuration space only and the compressing piston accesses the complementary portions of its configuration space.

The system will spontaneously fluctuate among the different stages. The probability of these fluctuations can be recovered from Einstein's analysis of fluctuations in a form summarized by Tolman (1938, pp. 637-38). The probability density $\mathrm{p}(\lambda)$ that the system will be found in stage $\lambda$ is proportional to the integral $Z(\lambda)$ :

$$
\mathrm{p}(\lambda)=\text { constant } \mathrm{Z}(\lambda)
$$

where the $Z(\lambda)$ is given by

$$
\mathrm{Z}(\lambda)=\int_{\lambda} \exp (-\mathrm{E}(\mathbf{x}, \boldsymbol{\pi}) / \mathrm{kT}) \mathrm{d} \mathbf{x} \mathrm{d} \boldsymbol{\pi}
$$

The integral extends over the subvolume of phase space corresponding to stage $\lambda$.

The essential idea behind the Einstein-Tolman analysis is to describe this stage $\lambda$ as if it were an equilibrium state, even though it can arise through a fluctuation. Then we can use the familiar results of equilibrium statistical thermodynamics to assign thermodynamic quantities to the state. Its partition function is $Z(\lambda)$. Each stage will also have a free energy $F(\lambda)=E(\lambda)-$ $\operatorname{TS}(\lambda)$ assigned to it, where $\mathrm{E}(\lambda)$ and $\mathrm{S}(\lambda)$ are the mean energy and the entropy also assigned to 
stage $\lambda$. For canonically distributed systems, this free energy is related to the partition function by

$$
F(\lambda)=-k T \ln Z(\lambda)
$$

Combining the last three equations, we have

$$
\mathrm{p}(\lambda)=\mathrm{C} \exp (-\mathrm{F}(\lambda) / \mathrm{kT})
$$

for $\mathrm{C}$ some constant; and the result that relates the probability density for the system fluctuating to states $\lambda_{1}$ and $\lambda_{2}$ :

$$
\mathrm{p}\left(\lambda_{2}\right) / \mathrm{p}\left(\lambda_{1}\right)=\exp \left(-\left(\mathrm{F}\left(\lambda_{2}\right)-\mathrm{F}\left(\lambda_{1}\right)\right) / \mathrm{kT}\right)
$$

The process is thermodynamically reversible. That means that, at each stage $\lambda$, the process satisfies the condition of thermal equilibrium on mean quantities. That is, $\mathrm{dF}(\lambda) / \mathrm{d} \lambda=$ $0,{ }^{14}$ so that $F(\lambda)$ is a constant in $\lambda$ and $F\left(\lambda_{2}\right)=F\left(\lambda_{1}\right)$. It now follows that $p\left(\lambda_{2}\right) / p\left(\lambda_{1}\right)=1$, so that $\mathrm{p}\left(\lambda_{2}\right)=\mathrm{p}\left(\lambda_{1}\right)$. In general, this means that

$$
\mathrm{p}(\lambda)=\text { constant, for all } \lambda
$$

That is, fluctuations are equally likely to carry the process from any stage to any other. If we try to set up the process in its initial stage, it is as likely to remain there as to fluctuate to any intermediate stage or the final stage. If the process has arrived at its final stage, it is as likely to remain there as to fluctuate back to any earlier stage.

A thermodynamically reversible process is required to proceed infinitely slowly. Hence, all efforts to realize this infinitely slow progress will be disrupted by these fluctuations. This completes the demonstration.

What does it take to bring the process reliably from start to end? It is easy to see that it requires entropy creation in excess of the $\mathrm{k} \ln 2$ of Landauer's Principle. For we need to create a gradient in the probability density that will favor completion. Since the probability density of the various stages $p(\lambda)$ are fixed by their free energies $F(\lambda)$, we need to reduce the free energy of the

14 If the process is in equilibrium at $\lambda$, the entropy of the system $S_{\text {sys }}$ and the entropy of the environment $S_{\text {env }}$ satisfy $(d / d \lambda)\left(S_{\text {sys }}(\lambda)+S_{\text {env }}(\lambda)\right)=0$. Since the system exchanges no work with the environment, but may exchange heat with it in a reversible process, we have $\mathrm{dS}_{\mathrm{env}}(\lambda) / \mathrm{d} \lambda=-$ $(1 / \mathrm{T}) \mathrm{dE}_{\text {sys }}(\lambda) / \mathrm{d} \lambda$. Combining we have $0=(\mathrm{d} / \mathrm{d} \lambda)\left(\mathrm{E}_{\mathrm{sys}}(\lambda)-\mathrm{T} . \mathrm{S}_{\mathrm{sys}}(\lambda)\right)=\mathrm{dF}_{\mathrm{sys}}(\lambda) / \mathrm{d} \lambda$. 
end stages. For example, we might introduce a disequilibrium over the process of $F\left(\lambda_{1}\right)>F\left(\lambda_{2}\right)$ $+3 \mathrm{kT}$, for which: 15

$$
\mathrm{p}\left(\lambda_{2}\right) / \mathrm{p}\left(\lambda_{1}\right)>\exp (3)=20
$$

This probability gradient discourages, but does not preclude, a fluctuation-driven reversion of the process from the end stage $\lambda_{2}$ to the initial stage $\lambda_{1}$. Nonetheless it comes at a heavy thermodynamic cost. Recalling that $\mathrm{F}=\mathrm{E}-\mathrm{TS}$, this disequilibrium is equivalent to

$$
\mathrm{S}\left(\lambda_{2}\right)-\mathrm{S}\left(\lambda_{1}\right)-\left(\mathrm{E}\left(\lambda_{2}\right)-\mathrm{E}\left(\lambda_{1}\right)\right) / \mathrm{T}>3 \mathrm{k}
$$

During this process, the energy change in the surroundings is $-\left(E\left(\lambda_{2}\right)-E\left(\lambda_{1}\right)\right)$. Hence the second term of this relation is a lower bound for the entropy increase in the surroundings. Thus, the relation asserts that the entropy of the system and its surroundings increases by at least $3 \mathrm{k}$ during the process. This entropy creation of $3 \mathrm{k}$ greatly exceeds the $\mathrm{k} \ln 2=0.69 \mathrm{k}$ of thermodynamic entropy that arises in Landauer's principle.

In sum, these results show us that all the reversible, isothermal processes we might seek to use in computation are fatally disrupted by fluctuations. We can overcome these fluctuations and drive the processes to completion only by introducing disequilibria that create quantities of entropy that greatly exceed those tracked by Landauer's Principle. This is catastrophic for the idea that entropy creation can be restricted ideally to erasure processes alone. The disequilibria needed to complete each step in the idealized computation will be creating quantities of entropy that will swamp the Landauer limit.

The problem only arises for processes that proceed at molecular scales. For ordinary, macroscopic processes, a disequilibrium corresponding to a $25 \mathrm{kT}$ difference in the free energy is negligible. This quantity of $25 \mathrm{kT}$ is the mean thermal energy of just ten oxygen molecules. However it is sufficient to produce a probability density ratio of $\exp (25)=7.2 \times 10^{10}$.

15 This modest intervention is quite violent. If we wish to encourage a one-molecule gas to expand to twice its volume, even removing the piston completely provides a free energy difference of merely $-\mathrm{kT} \ln 2=-0.69 \mathrm{kT}$. 


\subsection{An Illustration of the General Result}

The results of the last section are stated at a general level. To understand them better, it is helpful to instantiate the various quantities in an example. Consider the reversible compression or expansion of a one-molecule gas under a piston, as in Section 7.2. We will replace its gravitational force field by another field that acts only on the piston and not on the gas molecule. It exerts a downward force on the piston of magnitude $2 \mathrm{kT} / \mathrm{x}$, where $\mathrm{x}$ is the height of the piston above the cylinder floor. This particular force has been chosen since it will turn out that, under it, the piston can remain in equilibrium with the gas at all heights. Thus the energy of the piston will vary as

$$
\mathrm{E}_{\mathrm{pist}}(\mathbf{x}, \boldsymbol{\pi})=2 \mathrm{kT} \ln \mathrm{x}+\pi^{2} / 2 \mathrm{M},
$$

where $\mathrm{M}$ is the piston's mass and $\mathbf{x}=(\mathrm{x}, \mathrm{y}, \mathrm{z})$ and $\pi=\left(\pi_{\mathrm{x}}, \pi_{\mathrm{y}}, \pi_{\mathrm{z}}\right)$ are the piston's canonical position and momentum variables. The energy of the gas is

$$
\mathrm{E}_{\text {gas }}\left(\mathbf{x}^{\prime}, \pi^{\prime}\right)=\pi^{\prime 2} / 2 \mathrm{~m}
$$

where $m$ is the mass of its molecule and $\mathbf{x}^{\prime}=\left(\mathrm{x}^{\prime}, \mathrm{y}^{\prime}, \mathrm{z}^{\prime}\right)$ and $\pi^{\prime}=\left(\pi_{\mathrm{x}}{ }_{\mathrm{x}}, \pi^{\prime}{ }_{\mathrm{y}}, \pi^{\prime}{ }_{\mathrm{Z}}\right)$ are its canonical position and momentum variables.

When the compression or expansion is at stage $\mathrm{h}$, the system is restricted to the following subvolume of its phase space: the one-molecule gas occupies the region of the cylinder from $\mathrm{x}^{\prime}=0$ to $\mathrm{x}^{\prime}=\mathrm{h}$; and the piston fluctuates through the region $\mathrm{x}=\mathrm{h}$ to $\mathrm{x}=\infty .16$ The probability density $\mathrm{p}_{\mathrm{gas}}(\mathrm{h})$ that the gas extends to height $\mathrm{h}$ is proportional to the partition function

$$
\mathrm{Z}_{\mathrm{gas}}(\mathrm{h})=\int_{\mathrm{all}} \pi^{\prime}, \mathrm{y}^{\prime}, \mathrm{z}^{\prime} \int_{\mathrm{x}^{\prime}=0, \mathrm{~h}} \exp \left(-\pi^{\prime} / 2 \mathrm{mkT}\right) \mathrm{d} \mathbf{x}^{\prime} \mathrm{d} \boldsymbol{\pi}^{\prime}=\operatorname{const}(\mathrm{T}) . \int_{\mathrm{x}^{\prime}=0, \mathrm{~h}^{\prime}} \mathrm{dx} \mathrm{x}^{\prime}=\operatorname{const}(\mathrm{T}) \cdot \mathrm{h}
$$

The term const $(\mathrm{T})$ is a constant that is a function of $\mathrm{T}$ but not $\mathrm{h}$. Since differences between the constants that enter analogously into the formulae below will not affect the final result, I will use "const(T)" as a generic symbol for all such constants. The gas free energy is

$$
F_{\text {gas }}(h)=-k T \ln Z_{\text {gas }}(h)=-k T \ln h+\operatorname{const}(T)
$$

16 The interaction of the molecule and piston requires a short-range interaction term in the Hamiltonian that would ensure that the molecule bounces off the piston, rather than passing through it. This interaction is idealized here by the assumption that that the piston can never be found at a lower altitude than the molecule. 
The pressure force exerted by the gas is

$$
\mathrm{X}_{\text {gas }}(\mathrm{h})=-\partial /\left.\partial \mathrm{h}\right|_{\mathrm{T}} \mathrm{F}_{\mathrm{gas}}(\mathrm{h})=\mathrm{kT} / \mathrm{h}
$$

which is the force generated according to the ideal gas law by the pressure of a one-molecule gas.

The corresponding probability $\mathrm{p}_{\text {pist }}(\mathrm{h})$ for the piston is proportional to the partition function

$$
\begin{aligned}
Z_{\text {pist }}(h) & =\int_{\text {all } \pi, y, z} \int_{x=h, \infty} \exp \left(-\left(2 \mathrm{kT} \ln \mathrm{x}+\pi^{2} / 2 \mathrm{M}\right) / \mathrm{kT}\right) \mathrm{dxd} \pi \\
& =\operatorname{const}(\mathrm{T}) . \int_{\mathrm{x}=\mathrm{h}, \infty}\left(1 / \mathrm{x}^{2}\right) \mathrm{dx}=\operatorname{const}(\mathrm{T}) .(1 / \mathrm{h})
\end{aligned}
$$

The piston free energy is

$$
\mathrm{F}_{\text {piston }}(\mathrm{h})=-\mathrm{kT} \ln \mathrm{Z}_{\text {piston }}(\mathrm{h})=\mathrm{kT} \ln \mathrm{h}+\operatorname{const}(\mathrm{T})
$$

The mean force exerted by the piston on the gas is

$$
X_{\text {piston }}(h)=-\partial /\left.\partial h\right|_{T} F_{\text {piston }}(h)=-k T / h
$$

We can now see that the system is at equilibrium at all $\mathrm{h}$, for the mean forces exerted by the gas and piston balance

$$
\mathrm{X}_{\mathrm{gas}}(\mathrm{h})+\mathrm{X}_{\text {piston }}(\mathrm{h})=\mathrm{kT} / \mathrm{h}-\mathrm{kT} / \mathrm{h}=0
$$

Since $X(h)=-\partial /\left.\partial h\right|_{T} F(h)$, this is equivalent to requiring constancy of the sum of their free energies, $F_{\text {tot }}(\mathrm{h})$, which does obtain

$$
\mathrm{F}_{\text {tot }}(\mathrm{h})=\mathrm{F}_{\text {gas }}(\mathrm{h})+\mathrm{F}_{\text {piston }}(\mathrm{h})=-\mathrm{kT} \ln \mathrm{h}+\mathrm{kT} \ln \mathrm{h}+\operatorname{const}(\mathrm{T})=\operatorname{const}(\mathrm{T})
$$

Finally, we should expect the probability of the combined system to be uniformly distributed over all values of $h$. This also obtains, for the combined probability density $p_{\text {tot }}(h)$ is

$$
\mathrm{p}_{\text {tot }}(\mathrm{h})=\mathrm{p}_{\mathrm{gas}}(\mathrm{h}) \cdot \mathrm{p}_{\text {piston }}(\mathrm{h})=\operatorname{const}(\mathrm{T}) \cdot \mathrm{Z}_{\mathrm{gas}}(\mathrm{h}) \cdot \mathrm{Z}_{\text {piston }}(\mathrm{h})=\operatorname{const}(\mathrm{T}) \cdot \mathrm{h} \cdot(1 / \mathrm{h})=\operatorname{const}(\mathrm{T}) .
$$

Hence, through fluctuations, the system is equally likely to be at any stage of the compression or expansion process. This precludes the possibility of an infinitely slow expansion or contraction proceeding, as required by a reversible process.

The analysis of this case illustrates how these large fluctuations arise. Initially, it may seem odd that a gas-piston system, half expanded to stage $h=H / 2$, may fluctuate so freely to a fully expanded stage $\mathrm{h}=\mathrm{H}$. That oddness derives from a mistaken assumption about the 
microscopic dynamics. We expected erroneously that the dynamics would tend to keep the microstates within each stage, only slowly letting them evolve into later stages. However the dynamics allows the molecule and piston to move about so much in each stage that they are dynamically very close to and often coincide with the positions associated with other stages. Indeed, the phase volumes of the two stages overlap substantially. This is shown in Figure 6, which displays the equiprobable volumes corresponding to the various stages in configuration space.

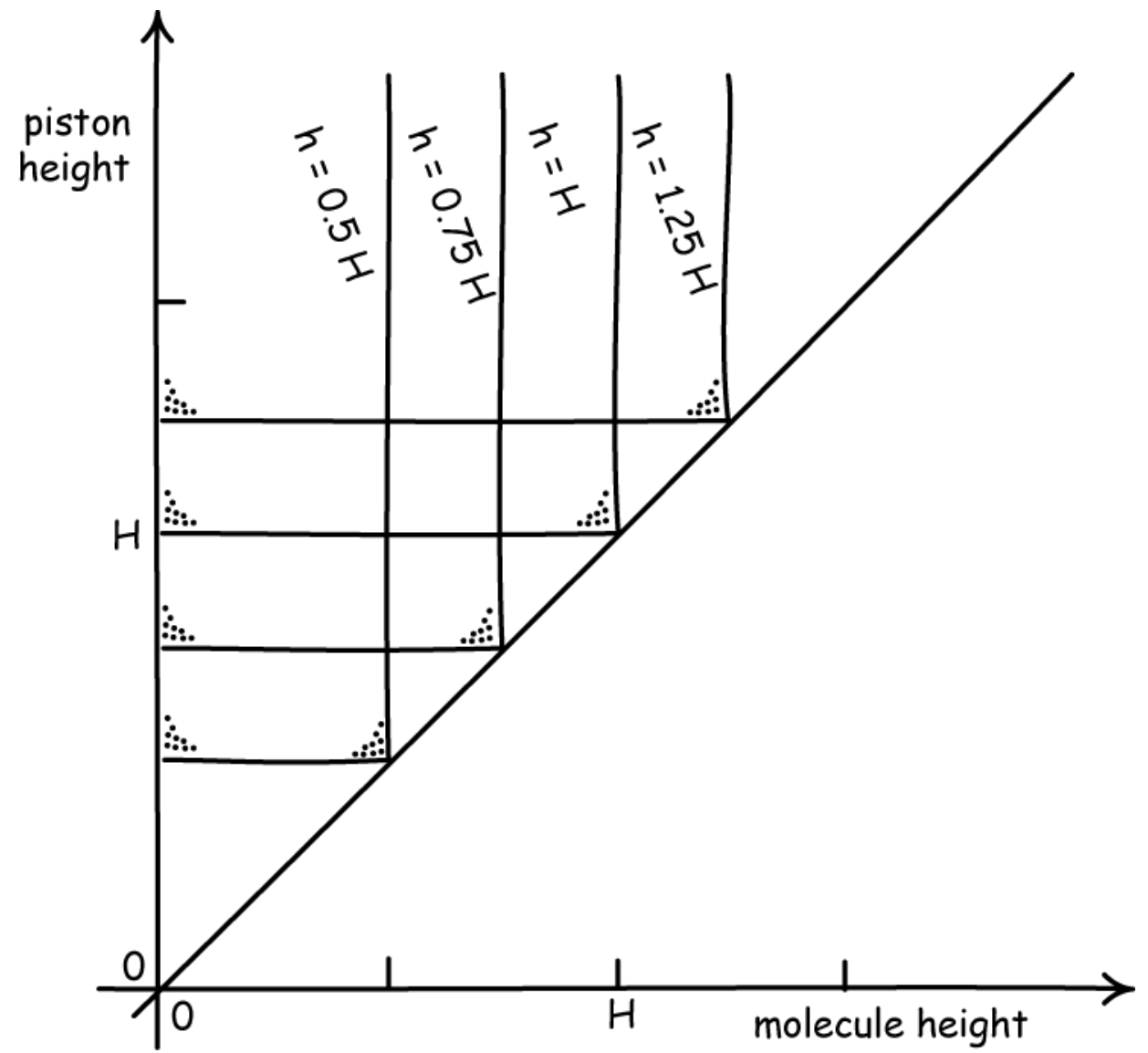

Figure 6. Configuration space for the gas-piston system

Thus, a microstate in stage $h=H / 2$ is as likely to evolve into another microstate in stage $h=H / 2$ as to a microstate in stage $\mathrm{h}=\mathrm{H}$. The apparent oddness of the ease of fluctuation derives from the artificiality of dividing up the system microstates into the stages of different $h$ values.

The moral is general. Figure 7 shows the phase space divided into subvolumes corresponding to the stages $\lambda$ of a general process. The representation on the left shows the expected time evolution in which the system remains for a longer time in each stage before 
passing to the next. The representation on the right shows what actually happens: the system bounces indifferently over all stages.
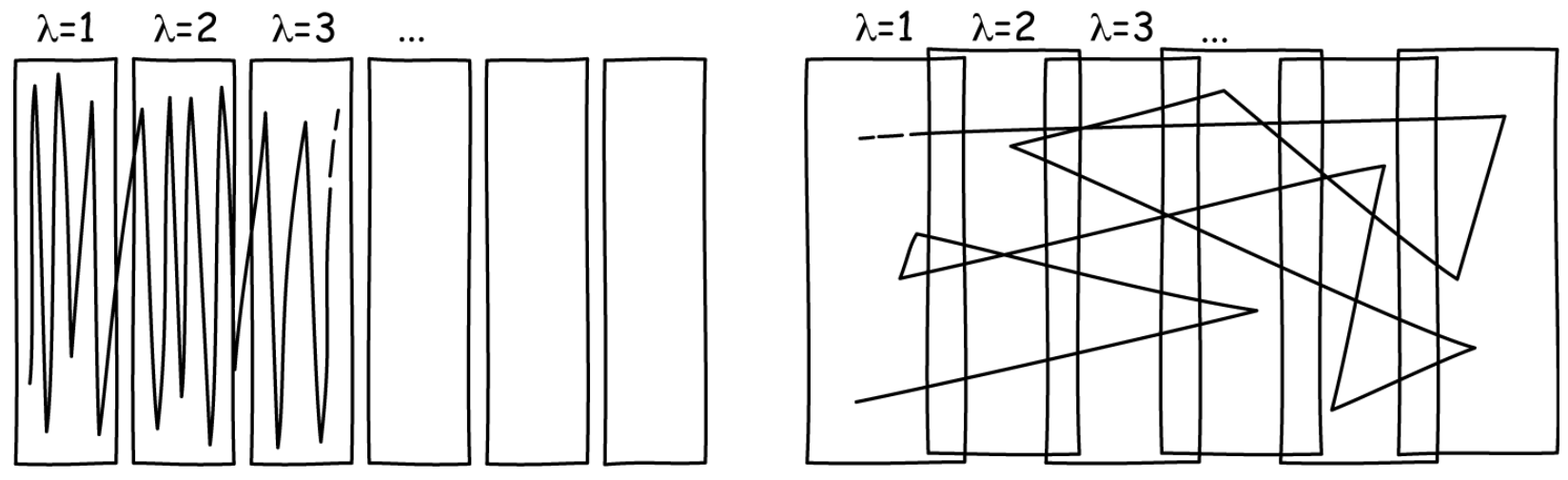

Figure 7. Intended and Actual Time Evolution for a Reversible Process

\subsection{Insertion of the Partition}

It is not hard to see that analogous problems will afflict the remaining processes once we try to make their details concrete. For example, Process $2 b$. Insertion of the partition, must terminate with the partition locked in place. But what arrests its motion? Since its physics is governed by a conservative Hamiltonian, what will prevent it bouncing off the stop at the end of its intended motion; and what will prevent fluctuations from rattling it loose from the mechanism that holds it? As before, a spring-loaded pin will fail. We cannot call up a tight fit into a groove, wedges, screws, nuts and bolts or ropes with knots, for all these devices depend upon friction and are incompatible with a conservative Hamiltonian. Perhaps the most promising approach is to set a very heavy weight over the partition. But now we must find a process that can deliver the weight to the right spot with no residual energy, else it will bounce off. As before, we cannot bleed off excess energy with friction, for that would be just the dissipative conversion of work to heat.

The analysis of this Section 7 overall show that we cannot construct an inventory of admissible microscopic processes by taking familiar macroscopic processes and assuming that they scale down to equally well-behaved microscopic processes. At ordinary scales, we may be able to build a delicately balanced house of cards on a tabletop. At microscopic scales, the tabletop is shuddering as if in a powerful earthquake and the cards are flapping about as if alive. 


\section{Assigning Thermodynamic Entropy to Probabilistic Mixtures}

\subsection{The Result Claimed and its Proof}

A sequel to LPSG seeks to revive the possibility of employing information theoretic entropy, as given by formula (3), as a way of assigning thermodynamic entropy. Ladyman et al. (2008) [LPS] consider the case in which the probabilities $P_{i}$ of formula (3) are distributed over distinct macrostates. The goal is to demonstrate that a statistical form of the second law entails that thermodynamic entropy must be attached to this probability distribution according to the information theoretic formula.

The result claimed and proof offered is presented at a greater level of generality than needed for the present analysis. So I will proceed as before by restricting both to the simple case of Section 4 above. The set-up will be the same, except that the one-molecule memory device $\mathrm{M}$ will be replaced by a huge cavern with a boulder that can be rolled to the left or right, where it occupies equal thermodynamic entropy macrostates $M_{L}$ and $M_{R}$. This device emphasizes the macroscopic character of the memory device. The proof proceeds by carrying out Steps 1-3. and then halting. At the end of Step 3., the system G has been returned to its original state. The totality of changes has been to pass heat kT $\ln 2$ to the heat bath and transform the boulder memory device from its initial macrostate $\mathrm{M}_{\mathrm{L}}$ to an equally weighted, probabilistic mixture of the two macrostates $M_{L}$ and $M_{R}$.

LPS follow the Landauer Principle literature in describing all the processes of Steps 1 to 3 as reversible, including the insertion of the partition. LPS now propose a definition of entropy that can assign a thermally based entropy to probabilistic mixtures of macrostates. For a reversible, isothermal process the entropy change $\Delta S$ is given by a version of Clausius' formula (1) adapted to the probabilistic environment

$$
\Delta \mathrm{S}=\left\langle\Delta \mathrm{q}_{\mathrm{rev}}>/ \mathrm{T}\right.
$$

where $<\Delta \mathrm{q}_{\mathrm{rev}}>$ is the mean quantity of heat passed to the system, assuming all heat flows are at one temperature $\mathrm{T}$.

In the course of the reversible process of Steps 1-3, the total system of G, M and the heat bath exchanges no heat with a larger system. If follows from (1') that the entropy of the total system remains constant. The heat bath has lost heat $\mathrm{kT} \ln 2$. By (1') its entropy has reduced by 
$(\mathrm{kT} \ln 2) / \mathrm{T}=\mathrm{k} \ln 2$. This reduction must be compensated by the only other component of the system that has changed, the boulder system $\mathrm{M}$, whose entropy must increase by $\mathrm{k} \ln 2$. That is, the boulder system $\mathrm{M}$ has gained an entropy of $\mathrm{k} \ln 2$ by virtue of being transformed into a probabilistic mixture of states, where the probabilities of the two states are $P_{L}=P_{R}=1 / 2$. This additional entropy turns to out to equal the information theoretic entropy computed by formula (3):

$$
\mathrm{S}_{\text {info }}=-\mathrm{k}\left(\mathrm{P}_{\mathrm{L}} \ln \mathrm{P}_{\mathrm{L}}+\mathrm{P}_{\mathrm{R}} \ln \mathrm{P}_{\mathrm{R}}\right)=\mathrm{k} \ln 2
$$

In sum, we have a boulder system probabilistically distributed over two positions $\mathrm{L}$ and $\mathrm{R}$ in a great cavern; and there is a thermodynamically defined entropy of $\mathrm{k} \ln 2$, computed by the information theoretic formula (3), associated with the probability distribution.

It is important to note that the proof of this result depends essentially on the holding of the statistical form of the second law of thermodynamics already mentioned in Section 4. For, by the argument sketched in Section 2.2 above, this law is necessary if the entropy differences among states and mixtures is to be independent of the reversible process connecting them. Only then will the entropy computed by formula (1') be a property of the probabilistic mixture, independent of the path taken by the process that created it.

\subsection{Why it Fails}

There are two difficulties, each sufficient to defeat the proof. The first is the same problem that affected the attempted indirect proof of Landauer's Principle. It employs an inventory of processes that is incompatible with the statistical form of the second law of thermodynamics. ${ }^{17}$ Hence, inferences to contradictory conclusions are possible in the system and the result is unreliable.

17 In the context of the LPS proof, we may challenge the inventory along lines similar to those indicated in Section 7. The inventory is supposed to include processes that can take fluctuations at the molecular level-the differing positions of a single molecule in a chamber - and amplify them into differences of macrostates. Of course an electron microscope can do this. But it does not operate reversibly in the thermodynamic sense. We are to suppose the amplification can be done reliably by thermodynamically reversible processes; that is, by processes that approach 
The second difficulty is the description of the process of insertion of the partition as "reversible." As I have argued at some length in Norton (2005, Section 3.2), this is a ruinous misuse of the term "reversible" if it is applied in the Clausius definition of thermodynamic entropy. The justification for the use of the term is, I believe, that inserting and removing the partition switches one between a fully expanded gas and a probabilistic mixture of states of the molecule trapped on one or other side of the partition. In that sense, each process is the reverse of the other. However it is the wrong sense of reversible if the entropy defined through the Clausius formula (1') is to be a state property.

To see why, consider a chamber holding a single molecule and insert a partition at the midpoint. We now have a one-molecule gas in the familiar probabilistic mixture of states $\mathrm{M}_{\mathrm{L}}$ and $\mathrm{M}_{\mathrm{R}}$. Drawing only on admissible processes in the inventory, we can construct two processes that restore the probabilistic mixture to the fully expanded state.

Expansion Process 1. We detect ${ }^{18}$ which side of the partition holds the molecule and insert a piston accordingly. We then perform a reversible, isothermal expansion of the one-molecule gas, so that the gas, in each case, absorbs heat kT $\ln 2$ from the heat bath. That quantity is the mean heat $\left\langle\Delta \mathrm{q}_{\mathrm{rev}}>\right.$ for formula (1'), from which we infer that the entropy difference between the mixture and the fully expanded state is $(\mathrm{kT} \ln 2) / \mathrm{T}=\mathrm{k} \ln$ 2.

Expansion Process 2. We remove the partition. The one-molecule gas expands to fill the chamber without any exchange of heat with the heat bath. If we concur with LPS that this is also a reversible process, then we can apply formula (1') and conclude that the mixture and the fully expanded state differ in entropy by $0 / \mathrm{T}=0$.

equilibrium processes arbitrarily closely. Should we not expect such processes to be fatally disrupted by thermal fluctuations in their own mechanisms?

18 That Process 1 only has a detection does not affect the argument since LPS assume that detection is possible reversibly and without passing heat to or from the gas. In any case, we can add a detection to the start of Process 2, even though its outcome is not exploited, to assure that they processes do not differ in this regard. 
It now follows that the entropy of $\left(1^{\prime}\right)$ is not a path-independent property, for we have two reversible processes that connect the same end points, but are associated with different entropy differences.

This mischaracterization of the insertion of the partition as a reversible process enables the proof to overlook the fact that the process is actually one that reduces the entropy of the onemolecule gas $\mathrm{G}$ by $\mathrm{k} \ln 2$. The omission of this reduction from the entropy accountancy leaves an entropy surfeit of $\mathrm{k} \ln 2$ that is then erroneously attributed to the probabilistic mixture.

\section{Conclusion}

We expect thermal problems to place limits on what we can achieve with computing machines. As the machines become smaller, the practical challenge of separating a computed signal from background thermal noise increases. When we try to make our computing machines thermodynamically more efficient by bringing their processes closer to thermodynamically reversible processes, this same thermal noise, manifested as fluctuations in the machinery, threatens to disrupt their intended operation.

It remains an open question, as far as I can see, whether these thermal problems are anything more than challenging practical problems whose effects can be minimized but never eliminated; or whether their limits can be reduced to some simple, sharp and principled expression. The present literature on the thermodynamics of computation has sought such a reduction by localizing these limits into just one type of process, logically irreversible computation of which erasure is a special case; and to give these limits principled expression as Landauer's Principle.

The central claims of this paper are that we still await a cogent justification of Landauer's Principle and that present efforts to demonstrate it are proceeding in an incoherent framework. Its proponents seek to assert both a statistical form of the second law of thermodynamics and Landauer's Principle. Yet the demonstrations associated with them employ a repertoire of processes through which both can be violated. It is an awkwardly constructed repertoire. Extraordinary attention is lavished on the thermal fluctuations of selected systems, most commonly, a single molecule in a chamber. That system is measured, expanded, contracted and more by a collection of further processes. Yet the inventory ignores the same thermal fluctuations in each component of these processes that threaten to defeat their operation. 
The inventory seems to have been constructed so that it can enable certain predetermined results to be established. We should like to see an entropy cost associated with erasure. So we focus on the thermal character of a single molecule whose position encodes our data. If we wish to restrict its position in an erasure operation, its thermal motions mean that, when we compress it, we pass heat to a reservoir creating thermodynamic entropy in the reservoir. But if we are interested only in reading our data by determining the molecule's position, we ignore the same thermal fluctuations in the processes used. Hence we preclude discovery of further thermodynamic costs that might compromise the core idea to be protected: that ineliminable dissipation only arises through processes that physically implement logically irreversible functions.

The concern that something is fundamentally awry in this literature has already been expressed in my earlier papers, including those with John Earman (Earman and Norton, 1998, 1999; Norton 2005). We found a literature based on unsound principles and methods. More recent work in that literature has not improved matters. It takes that same unsound foundation and adds layers of more elaborate theorizing. Those efforts may produce analysis that is rigorous and even ingenious in its smaller parts. However the totality remains incoherent, with its unsound foundations now obscured by the sheer mass of the new theorizing. 


\section{Appendix: Some Recent Attempts to Prove Landauer's Principle}

\section{By An Illicit Canonical Ensemble}

Piechocinska's (2000) considers the erasure process for three analogous systems, arriving at Landauer's Principle for each. Here I will consider the first system. In it, the memory device is modeled as classical particle trapped in one side of a symmetric double well potential field. The device is in thermal equilibrium with a heat reservoir at temperature $\mathrm{T}$ throughout the erasure process. In the initial state, the molecule is equally likely to be trapped in either well and its probability density in its phase space is $\rho_{\text {init }}$. During the erasure process, work is supplied to the memory device and a quantity of heat $\Delta \mathrm{E}$ is passed to the reservoir. The device with its particle in the reset state then has a probability density $\rho_{\text {final }}$.

A principal burden of the analysis is to derive Piechocinska's equation (9):

$$
<\ln \rho_{\text {final }}>-<\ln \rho_{\text {init }}>\leq<\Delta \mathrm{E}>/ \mathrm{kT}
$$

where angle brackets represent phase averages. This inequality will likely appear unfamiliar until one recognizes the quantities. Since the molecule is canonically distributed, we have

$$
\rho=\exp (-\mathrm{E}(\mathrm{x}) / \mathrm{kT}) / \mathrm{Z}
$$

where $\mathrm{E}$ is the energy of the molecule when at generalized phase space coordinate $\mathrm{x} . \mathrm{Z}$ is the normalizing partition function

$$
Z=\int \exp (-E(x) / k T) d x
$$

Thus

$$
<\ln \rho>=-<\mathrm{E}>/ \mathrm{kT}-\ln \mathrm{Z}
$$

which is merely a rescaled expression of the canonical thermodynamic entropy S of the Gibbs approach:

$$
\mathrm{S}=-\mathrm{k}<\ln \rho>
$$

Hence inequality (A1) asserts a relation familiar in the Gibbs approach and in no need of a new derivation. For an isothermal process

$$
\mathrm{S}_{\text {final }}-\mathrm{S}_{\text {init }} \geq-<\Delta \mathrm{E}>/ \mathrm{T}
$$


where $-<\Delta \mathrm{E}>$ is the heat supplied to the system. In words, the entropy increase of a system in an isothermal process is at least equal to (heat gained)/T. Equality obtains for a reversible process.

All that remains is to show that the erasure process passes heat $\langle\Delta \mathrm{E}\rangle=\mathrm{k} \ln 2$ to the heat reservoir thereby creating thermodynamic entropy $\mathrm{k} \ln 2$. To arrive at this, we note that the single particle before and after the process has the same mean energy, $\left\langle\mathrm{E}_{\text {init }}\right\rangle=\left\langle\mathrm{E}_{\text {final }}\right\rangle$. Hence

$$
<\ln \rho_{\text {final }}>-<\ln \rho_{\text {init }}>=\ln Z_{\text {init }}-\ln Z_{\text {final }}=\ln \left(Z_{\text {init }} / Z_{\text {final }}\right)
$$

Everything in this derivation thus far is correct. Now the error enters. Piechocinska presumes (p. 1) an "ensemble of bits" with "half the bits to be in the 'one' state and the other half to be in the 'zero' state." This initial state is represented by a canonical probability distribution that is spread over both wells of the double potential well, even though each particle can access only one of the wells. Since the double wells are symmetric in their phase spaces, it then follows that

$$
\mathrm{Z}_{\text {init }}=\int_{\text {two wells }} \exp (-\mathrm{E}(\mathrm{x}) / \mathrm{kT}) \mathrm{dx}=2 \mathrm{Z}_{\text {final }}=2 \int_{\text {one well }} \exp (-\mathrm{E}(\mathrm{x}) / \mathrm{kT}) \mathrm{dx}
$$

Hence $\ln \left(\mathrm{Z}_{\text {init }} / \mathrm{Z}_{\text {final }}\right)=2$. Combining we conclude that the heat passed to the reservoir, $<\mathrm{E}>$, is greater than or equal to kT $\ln 2$ as Landauer's Principle requires.

The error of this proof is to represent the ensemble of bits carrying equally many zeros and ones by a canonical distribution spread over both wells. It is precisely the illicit canonical ensemble described above in Section 3.3. The canonical distribution spread over two wells represents the memory devices after they have been thermalized so that the particles can access both wells and, as a result, each device's thermodynamic entropy has been increased by k $\ln 2$.

The correct analysis represents the initial state of each device individually by a canonical distribution restricted to the relevant well. As a result, for each device we have $Z_{\text {init }}=Z_{\text {final }}$ and the proof can no longer assure that heat must be passed to the reservoir since in erasure, since (A1) reduces to $0 \leq<\Delta \mathrm{E}>$.

\section{By Compression of Phase Volume}

Turgut's (2009) attempted demonstration of Landauer's Principle is a sophisticated and, in places, elegant, version of the direct proofs that represent erasure as a compression of the phase space. Landauer's Principle arises as a special case in the analysis of stochastic processes in the statistical physics of Hamiltonian systems. The input to the process is a system in 
equilibrium with a heat bath. The system's phase space is divided into cells labeled $\alpha=1, \ldots, \mathrm{n}$, one of which is occupied by the system point. The process transforms this to a new system in equilibrium with a heat bath. The new system phase space is divided into cells labeled $\beta=1, \ldots$, m. $\mathrm{P}(\beta \mid \alpha)$ is the probability of a transition from initial state $\alpha$ to final state $\beta$. The case of erasure arises if we regard the system as a memory device and take a transformation that assuredly sends all input states $\alpha$ to a nominated reset state, $\beta=1$. That is, $P(\beta \mid \alpha)=\delta_{\beta 1}$.

The generality of Turgut's result makes the proof very complicated and, as a result, difficult to follow. So I will restrict discussion here to the simplest case of erasure for which the basic of idea of the proof is easily seen. In that simple case, the initial and final systems are the same system $\mathrm{S}$ and their phase space is divided into the same set of $\mathrm{n}$ identical cells. They are at equilibrium with the same heat bath $\mathrm{B}$ at the same temperature $\mathrm{T}$. The process is effected by performing work on the system. For example, the phase space may be divided into cells by walls comprising fields. Alterations in these fields correspond to raising, lowering or moving of these walls. These changes are represented formally by the time dependence of the total system Hamiltonian.

The origin of Landauer's Principle is explained in terms of phase space compression (p.1):

LEP [Landauer's Principle] follows from the constancy of the phase space measures: as the process necessarily reduces the phase space of the device by requirement (B) [that requires the process to reset the memory device], it must expand that of the environment, which leads to the Landauer bound.

That erasure compresses the phase space is not posited directly but is derived from a further assumption upon which the paper based (p. 1):

(A) The process is carried out in the same way independent of the initial logical state

(i.e. the information stored) or the microstate of the device...

This informal conditions is rendered more precisely as (p. 1)

...feature (A) is equivalent to the statement that the time dependence of the microstate during the process is governed by a single logical-state-independent Hamiltonian.

In the special case of erasure, this condition requires a process that compresses the phase space of all the $\mathrm{n}$ input states $\alpha=1, \ldots, \mathrm{n}$ to the single cell $\beta=1$ of the final reset device. This follows 
since we do not know which input cell will contain the system point, but the time evolution has to be such that, whichever cell that may be, the system will end up in the reset state $\beta=1$. So the Hamiltonian flow must map all n cells - "many-to-one"-into the single reset cell. Since the time evolution is Hamiltonian, Liouville's theorem applies and total phase volume is conserved. The compression of phase volume in the phase space of the memory device $\mathrm{S}$ is compensated by an increase in the phase volume of the heat bath B that is at least as great. Hence: 19

$$
\begin{array}{cll}
\text { Sum of phase volume for } & & \text { Phase volume of reset } \\
\text { all possible input states of } & \leq & \text { cell in S, including } \\
\mathrm{S} \text {, including heat bath } \mathrm{B} . & & \text { heat bath B. }
\end{array}
$$

In the course of the process, some amount of work $\mathrm{W}$ will be communicated to the system $\mathrm{S}$ and an amount of heat $\mathrm{Q}$ will be passed from $\mathrm{S}$ to the heat bath $\mathrm{B}$ at temperature T. For this simplified case, the initial cell $\alpha$ and the final cell $\beta=1$ are assumed to be identical in physical properties. Hence their mean energies are the same. So we have $Q=W$. The entropy s (in units of Boltzmann's constant k) created by the erasure process is due to this heat Q passed to the heat bath $\mathrm{B}$ and is

$$
\mathrm{ks}=\mathrm{Q} / \mathrm{T}=\mathrm{W} / \mathrm{T}
$$

Since all memory device cells are the same physically, this entropy will be the same no matter which memory state is erased.

The phase volume $\mathrm{n}_{\mathrm{B}}(\mathrm{E})$ of the heat bath $\mathrm{B}$ will vary with its total energy. We set this energy at $\mathrm{E}$ initially and it becomes $\mathrm{E}+\mathrm{W}$ after the heat bath has gained heat $\mathrm{Q}=\mathrm{W}$. This increase in phase volume corresponds to the entropy created by erasure. If the phase volume of the input cells are each $\mathrm{Z}_{\mathrm{i \alpha}}$ and of the single reset cell is $\mathrm{Z}_{\mathrm{f} 1}$, the above condition on phase volumes becomes

$$
\sum_{\alpha} n_{B}(E) Z_{i \alpha} \leq n_{B}(E+W) Z_{f 1}
$$

19 This condition is a simplification of Turgut's equation (20), p. 4, whose positing is the essential step in the proof of Turgut's Theorem 1. Liouville's Theorem would require exact equality for the special case of erasure described, since the mapping of states is "onto." I retain the inequality of the general case for consistency with Turgut's text. 
Since the initial and final cells are the same in physical properties, we have $Z_{i \alpha}=Z_{f 1}$ for each $\alpha$. For a large heat bath of many components with $\mathrm{W}$ very much less than $\mathrm{E}$, so that $\mathrm{T}$ remains unchanged, we have

$$
\mathrm{n}_{\mathrm{B}}(\mathrm{E}+\mathrm{W})=\mathrm{n}_{\mathrm{B}}(\mathrm{E}) \exp (\mathrm{W} / \mathrm{kT})
$$

Combining and cancelling equal quantities we have

$$
\mathrm{n} \leq \exp (\mathrm{W} / \mathrm{kT})
$$

Recalling that $\mathrm{s}=\mathrm{W} / \mathrm{k}$ we arrive at the principal result of Turgut's Theorem 1 (p. 1), specialized to the case at hand

$$
\mathrm{n} \exp (-\mathrm{s}) \leq 1
$$

Rewritten in a more familiar form, it tells us that erasing a memory device with $\mathrm{n}$ cells produces entropy ks that is no less than $\mathrm{k} \ln \mathrm{n}$

Promising as this demonstration may seem, it fails to establish that erasing the $\mathrm{n}$ state memory device necessarily creates at least $\mathrm{k} \ln \mathrm{n}$ of thermodynamic entropy. The proof does not establish that erasure must compress the memory device phase space. The initial and final states occupy the same phase volume, that of a single occupied cell. Thus erasure need only rearrange the phase space volumes, not compress them. Turgut argues otherwise on the basis of condition (A), which is unnecessarily restrictive. It is justified by a familiar argument (p.1):

Although it is conceivable that the process can read the information and take different actions depending on it, this can be done only by recording the information somewhere else. In that case, the process must also erase the recorded information.

This mistaken view persists, as far as I can see, because it is easy to anthropomorphize the erasure device as a little man who must always record what he is doing and then erase his records at the end. Absent that anthropomorphism, it is hard to see how the mistake can be sustained.

Similar to the suggestion of the main text (Section 6.2), one might imagine that each memory cell is attached to its own erasure device. To erase, all the devices are activated. Only one, the $\alpha$-device attached to cell $\alpha$, is triggered by its detection that the input state is $\alpha$. The remaining devices detect nothing and do nothing. Then independently of whether that triggering data continues to exist, the $\alpha$-device mechanically executes its sole function, the erasure routine specifically tailored to cell $\alpha$. It is programmed to terminate in its initial state automatically once its steps are completed. It has no memory registers that replicate the data of cell $\alpha$. It has no need of them. It does the same thing whether the cell to which it is attached is one of ten cells or one 
of a thousand cells. When triggered, it goes blindly through the action of moving the content of cell $\alpha$ to cell $\beta=1$.

\section{References}

Albert, D. (2000). Time and Chance. Cambridge, MA: Harvard University Press.

Bennett, Charles (1982) “The Thermodynamics of Computation-A Review,” International Journal of Theoretical Physics, 21, pp. 905-40; reprinted in Leff and Rex, 2003, Ch. 7.1.

Bennett, Charles, H. (1987) “Demons, Engines and the Second Law,” Scientific American, 257(5), pp. 108-116.

Bennett, Charles H. (2003) “Notes on Landauer's Principle, Reversible Computation, and Maxwell's Demon," Studies in History and Philosophy of Modern Physics, 34, pp. 50110 .

Earman, John and Norton, John D. (1998), "Exorcist XIV: The Wrath of Maxwell's Demon." Part I "From Maxwell to Szilard," Studies in the History and Philosophy of Modern Physics, 29(1998), pp.435-471.

Earman, John and Norton, John D. (1999), "Exorcist XIV: The Wrath of Maxwell's Demon." Part II: "From Szilard to Landauer and Beyond," Studies in the History and Philosophy of Modern Physics, 30(1999), pp.1-40.

Feynman, R.; Leighton, R. and Sands, M. The Feynman Lectures on Physics, Vol. 1. Reading,MA: Addison-Wesley.

[LPSG] Ladyman, James, Presnell, Stuart, Short, Anthony J., Groisman, Berry (2007), “The connection between logical and thermodynamic irreversibility," Studies in the History and Philosophy of Modern Physics, 38, pp. 58-79.

[LPS] Ladyman, J., Presnell, S., and Short, A. (2008), 'The Use of the Information-Theoretic Entropy in Thermodynamics', Studies in History and Philosophy of Modern Physics, 39, pp. 315-324.

Landauer, Rolf, (1961), "Irreversibility and heat generation in the computing process", IBM Journal of Research and Development, 5: 183-191; reprinted in Leff and Rex (2003, Ch. 4.1).

Landauer, Rolf (1993) "Information is Physical," Proceedings of PhysComp 1992. IEEE Computer Society Press. 
Leff, Harvey S. and Rex, Andrew (2003), eds., Maxwell's Demon 2: Entropy, Classical and Quantum Information, Computing. Bristol and Philadelphia: Institute of Physics Publishing.

Maroney, Owen (2005), "The (absence of a) relationship between thermodynamic and logical irreversibility", Studies in the History and Philosophy of Modern Physics, 36, pp. 355374.

Maroney, Owen (2009) "Information Processing and Thermodynamic Entropy", The Stanford Encyclopedia of Philosophy (Fall 2009 Edition), Edward N. Zalta (ed.), URL = $<$ http://plato.stanford.edu/archives/fall2009/entries/information-entropy/>.

Norton, John D. (2005), "Eaters of the lotus: Landauer's principle and the return of Maxwell's demon", Studies in the History and Philosophy of Modern Physics, 36, pp. 375-411.

Norton, John D. (2006),"Atoms Entropy Quanta: Einstein's Miraculous Argument of 1905," Studies in History and Philosophy of Modern Physics, 37, pp. 71-100.

Norton, John D. (2008) "Ignorance and Indifference," Philosophy of Science, 75, pp. 45-68.

Norton, John D. (2010), "Cosmic Confusions: Not Supporting versus Supporting Not-". Philosophy of Science, 77, pp. 501-523

Piechocinska, Barbara (2000) "Information Erasure," Physical Review A, 61, 062314, pp. 1-9.

Shenker, Orly (1999), "Maxwell's Demon and Baron Munchausen: Free will as a perpetuum mobile," Studies in History and Philosophy of Modern Physics, 30, pp. 347-372.

Shenker, Orly and Hemmo, Meir (2006), "Maxwell's Demon," http://philsci-archive.pitt.edu /archive/00003795/.

Szilard, Leo, (1929), "Uber die Entropieverminderung in einem thermodynamischen System bei Eingriffen intelligenter Wesen [On the Decrease of Entropy in a Thermodynamic System by the Intervention of Intelligent Beings]", Zeitschrift für Physik, 5, pp. 840-856.

Tolman, Richard C. (1938) The Principles of Statistical Mechanics. London: Oxford University Press.

Turgut, S. (2009) "Relations between Entropies Produced in Non-deterministic Thermodynamic Processes" Physical Review E, 79, 41102, pp. 1-11. 